\title{
IMPLICIT RENEWAL THEORY AND POWER TAILS ON TREES
}

\author{
PREDRAG R. JELENKOVIĆ* ${ }^{*}$ AND \\ MARIANA OLVERA-CRAVIOTO, ${ }^{* *}$ Columbia University
}

\begin{abstract}
We extend Goldie's (1991) implicit renewal theorem to enable the analysis of recursions on weighted branching trees. We illustrate the developed method by deriving the powertail asymptotics of the distributions of the solutions $R$ to $R \stackrel{\mathrm{D}}{=} \sum_{i=1}^{N} C_{i} R_{i}+Q, R \stackrel{\mathrm{D}}{=}$ $\left(\bigvee_{i=1}^{N} C_{i} R_{i}\right) \vee Q$, and similar recursions, where $\left(Q, N, C_{1}, C_{2}, \ldots\right)$ is a nonnegative random vector with $N \in\{0,1,2,3, \ldots\} \cup\{\infty\}$, and $\left\{R_{i}\right\}_{i \in \mathbb{N}}$ are independent and identically distributed copies of $R$, independent of $\left(Q, N, C_{1}, C_{2}, \ldots\right)$; here ' $\vee$ ' denotes the maximum operator.
\end{abstract}

Keywords: Implicit renewal theory; weighted branching process; multiplicative cascade; stochastic recursion; power law; large deviations; stochastic fixed-point equation

2010 Mathematics Subject Classification: Primary 60H25

Secondary 60J80; 60F10; 60K05

\section{Introduction}

This paper is motivated by the study of the nonhomogeneous linear recursion

$$
R \stackrel{\mathrm{D}}{=} \sum_{i=1}^{N} C_{i} R_{i}+Q
$$

where $\left(Q, N, C_{1}, C_{2}, \ldots\right)$ is a nonnegative random vector with $N \in \mathbb{N} \cup\{\infty\}, \mathbb{N}=\{0,1,2$, $3, \ldots\}, \mathrm{P}(Q>0)>0$, and $\left\{R_{i}\right\}_{i \in \mathbb{N}}$ is a sequence of independent and identically distributed (i.i.d.) random variables, independent of $\left(Q, N, C_{1}, C_{2}, \ldots\right)$, with the same distribution as $R$. This recursion appeared recently in the stochastic analysis of Google's PageRank algorithm; see [19], [27], and the references therein for the latest work in this area. These types of weighted recursions, also studied in the literature on weighted branching processes [25] and branching random walks [8], are found in the probabilistic analysis of other algorithms as well [24], [26], e.g. the Quicksort algorithm [13].

In order to study the preceding recursion in its full generality, we extend the implicit renewal theory of Goldie [14] to cover recursions on trees. The extension of Goldie's theorem is presented in Theorem 3.1. One of the observations that allows this extension is that an appropriately constructed measure on a weighted branching tree is a renewal measure; see Lemma 3.1 and (3.4). In the remainder of the paper we apply the newly developed framework

Received 1 February 2011; revision received 15 October 2011.

* Postal address: Department of Electrical Engineering, Columbia University, New York, NY 10027, USA.

Supported by the NSF, grant no. CMMI-1131053.

** Postal address: Department of Industrial Engineering and Operations Research, Columbia University, New York, NY 10027, USA. Email address: molvera@ieor.columbia.edu

Supported by the NSF, grant no. CMMI-1131053. 
to analyze a number of linear and nonlinear stochastic recursions on trees, starting with (1.1). Note that the majority of the work in the rest of the paper addresses the application of the main theorem to specific problems.

In this regard, in Section 4, we first construct an explicit solution (4.6) to (1.1) on a weighted branching tree, and then provide sufficient conditions for the finiteness of moments and the uniqueness of this solution in Lemmas 4.4 and 4.5, respectively. Furthermore, it is worth noting that our moment estimates are explicit (see Lemma 4.3), which may be of independent interest. Then, the main result, which characterizes the power-tail behavior of $R$ is presented in Theorem 4.1. In addition, for an integer power exponent $(\alpha \in\{1,2,3, \ldots\})$, the asymptotic tail behavior can be explicitly computed as stated in Corollary 4.1. Furthermore, for a noninteger $\alpha$, Lemma 4.1 yields an explicit bound on the tail behavior of $R$. Related work in the literature of weighted branching processes (WBPs) for the case when $N=\infty$ and $Q,\left\{C_{i}\right\}$ are nonnegative deterministic constants can be found in [25] (see Theorem 5), and, more recently, for real-valued constants, in [4]. However, these deterministic assumptions fall outside the scope of this paper; for more details, see the remarks after Theorem 4.1 in Section 4.2.

Next, we show how our technique can be applied to study the tail asymptotics of the solution to the critical, $\mathrm{E}\left[\sum_{i=1}^{N} C_{i}\right]=1$, homogeneous linear equation

$$
R \stackrel{\mathrm{D}}{=} \sum_{i=1}^{N} C_{i} R_{i}
$$

where $\left(N, C_{1}, C_{2}, \ldots\right)$ is a nonnegative random vector with $N \in \mathbb{N} \cup\{\infty\}$ and $\left\{R_{i}\right\}_{i \in \mathbb{N}}$ is a sequence of i.i.d. random variables independent of $\left(N, C_{1}, C_{2}, \ldots\right)$ with the same distribution as $R$. This type of recursion has been studied to a great extent under a variety of names, including branching random walks and multiplicative cascades. Our work is more closely related to the results of [17] and [23], where the conditions for power-tail asymptotics of the distribution of $R$ with power exponent $\alpha>1$ were derived. In Theorem 4.2 we provide alternative derivations of Theorem 2.2 of [23] and Proposition 7 of [17]. Furthermore, we note that our method yields a more explicit characterization of the power-tail proportionality constant; see Corollary 4.2. For a full description of the set of solutions to (1.2), see the recent work in [6]. For additional references on weighted branching processes and multiplicative cascades, see [2], [22]-[24], [28], and the references therein. For earlier historical references, see [12], [16], and [20].

As an additional illustration of the newly developed framework, in Section 5 we study the recursion

$$
R \stackrel{\mathrm{D}}{=}\left(\bigvee_{i=1}^{N} C_{i} R_{i}\right) \vee Q,
$$

where $\left(Q, N, C_{1}, C_{2}, \ldots\right)$ is a nonnegative random vector with $N \in \mathbb{N} \cup\{\infty\}, \mathrm{P}(Q>0)>0$, and $\left\{R_{i}\right\}_{i \in \mathbb{N}}$ is a sequence of i.i.d. random variables independent of $\left(Q, N, C_{1}, C_{2}, \ldots\right)$ with the same distribution as $R$. We characterize the tail behavior of $\mathrm{P}(R>x)$ in Theorem 5.1. Similarly to the homogeneous linear case, this recursion was previously studied in [5] under the assumptions that $Q \equiv 0, N=\infty$, and the $\left\{C_{i}\right\}$ are real-valued deterministic constants. The more closely related case of $Q \equiv 0$ and $\left\{C_{i}\right\} \geq 0$ being random was studied earlier in [18]. Furthermore, these max-type stochastic recursions appear in a wide variety of applications, ranging from the average case analysis of algorithms to statistical physics; see [1] for a recent survey. 
We conclude the paper with a brief discussion of other nonlinear recursions that could be studied using the developed techniques, including the solution to

$$
R \stackrel{\mathrm{D}}{=}\left(\bigvee_{i=1}^{N} C_{i} R_{i}\right)+Q .
$$

The majority of the proofs are postponed to Section 7.

\section{Model description}

First we construct a random tree $\mathcal{T}$. We use the notation $\varnothing$ to denote the root node of $\mathcal{T}$ and $A_{n}, n \geq 0$, to denote the set of all individuals in the $n$th generation of $\mathcal{T}, A_{0}=\{\varnothing\}$. Let $Z_{n}$ be the number of individuals in the $n$th generation, that is, $Z_{n}=\left|A_{n}\right|$, where $|\cdot|$ denotes the cardinality of a set; in particular, $Z_{0}=1$.

Next, let $\mathbb{N}_{+}=\{1,2,3, \ldots\}$ be the set of positive integers and let $U=\bigcup_{k=0}^{\infty}\left(\mathbb{N}_{+}\right)^{k}$ be the set of all finite sequences $\boldsymbol{i}=\left(i_{1}, i_{2}, \ldots, i_{n}\right)$, where, by convention, $\mathbb{N}_{+}^{0}=\{\varnothing\}$ contains the null sequence $\varnothing$. To ease the exposition, for a sequence $\boldsymbol{i}=\left(i_{1}, i_{2}, \ldots, i_{k}\right) \in U$, we write $\boldsymbol{i} \mid n=\left(i_{1}, i_{2}, \ldots, i_{n}\right)$, provided $k \geq n$, and $\boldsymbol{i} \mid 0=\varnothing$ to denote the index truncation at level $n, n \geq 0$. Also, for $i \in A_{1}$, we simply use the notation $i=i_{1}$, that is, without the parentheses. Similarly, for $\boldsymbol{i}=\left(i_{1}, \ldots, i_{n}\right)$, we will use $(\boldsymbol{i}, j)=\left(i_{1}, \ldots, i_{n}, j\right)$ to denote the index concatenation operation; if $i=\varnothing$ then $(i, j)=j$.

We iteratively construct the tree as follows. Let $N$ be the number of individuals born to the root node $\varnothing, N_{\varnothing}=N$, and let $\left\{N_{i}\right\}_{i \in U}$ be i.i.d. copies of $N$. Now define

$$
\begin{aligned}
& A_{1}=\left\{i \in \mathbb{N}_{+}: 1 \leq i \leq N\right\} \\
& A_{n}=\left\{\left(i_{1}, i_{2}, \ldots, i_{n}\right) \in U:\left(i_{1}, \ldots, i_{n-1}\right) \in A_{n-1}, 1 \leq i_{n} \leq N_{\left(i_{1}, \ldots, i_{n-1}\right)}\right\} .
\end{aligned}
$$

It follows that the number of individuals $Z_{n}=\left|A_{n}\right|$ in the $n$th generation, $n \geq 1$, satisfies the branching recursion

$$
Z_{n}=\sum_{i \in A_{n-1}} N_{i}
$$

Now, we construct the weighted branching tree $\mathcal{T}_{Q, C}$ as follows. The root node $\varnothing$ is assigned a vector $\left(Q_{\varnothing}, N_{\varnothing}, C_{(\varnothing, 1)}, C_{(\varnothing, 2)}, \ldots\right)=\left(Q, N, C_{1}, C_{2}, \ldots\right)$ with $N \in \mathbb{N} \cup\{\infty\}$ and $\mathrm{P}(Q>$ $0)>0 ; N$ determines the number of nodes in the first generation of $\mathcal{T}$ according to (2.1). Each node in the first generation is then assigned an i.i.d. copy $\left(Q_{i}, N_{i}, C_{(i, 1)}, C_{(i, 2)}, \ldots\right)$ of the root vector and the $\left\{N_{i}\right\}$ are used to define the second generation in $\mathcal{T}$ according to (2.1). In general, for $n \geq 2$, to each node $i \in A_{n-1}$, we assign an i.i.d. copy $\left(Q_{i}, N_{i}, C_{(i, 1)}, C_{(i, 2)}, \ldots\right)$ of the root vector and construct $A_{n}=\left\{\left(\boldsymbol{i}, i_{n}\right) \in U: \boldsymbol{i} \in A_{n-1}, 1 \leq i_{n} \leq N_{i}\right\}$; the vectors $\left(Q_{i}, N_{i}, C_{(i, 1)}, C_{(i, 2)}, \ldots\right), i \in A_{n-1}$, are chosen independently of all the previously assigned vectors $\left(Q_{\boldsymbol{j}}, N_{\boldsymbol{j}}, C_{(\boldsymbol{j}, 1)}, C_{(\boldsymbol{j}, 2)}, \ldots\right), \boldsymbol{j} \in A_{k}, 0 \leq k \leq n-2$. For each node in $\mathcal{T}_{Q, C}$, we also define the weight $\Pi_{\left(i_{1}, \ldots, i_{n}\right)}$ via the recursion

$$
\Pi_{i_{1}}=C_{i_{1}}, \quad \Pi_{\left(i_{1}, \ldots, i_{n}\right)}=C_{\left(i_{1}, \ldots, i_{n}\right)} \Pi_{\left(i_{1}, \ldots, i_{n-1}\right)}, \quad n \geq 2,
$$

where $\Pi=1$ is the weight of the root node. Note that the weight $\Pi_{\left(i_{1}, \ldots, i_{n}\right)}$ is equal to the product of all the weights $C_{(\cdot)}$ along the branch leading to node $\left(i_{1}, \ldots, i_{n}\right)$, as depicted in Figure 1. In some places, e.g. in the following section, the value of $Q$ may be of no importance, and, thus, we will consider a weighted branching tree defined by the smaller vector $\left(N, C_{1}, C_{2}, \ldots\right)$. 


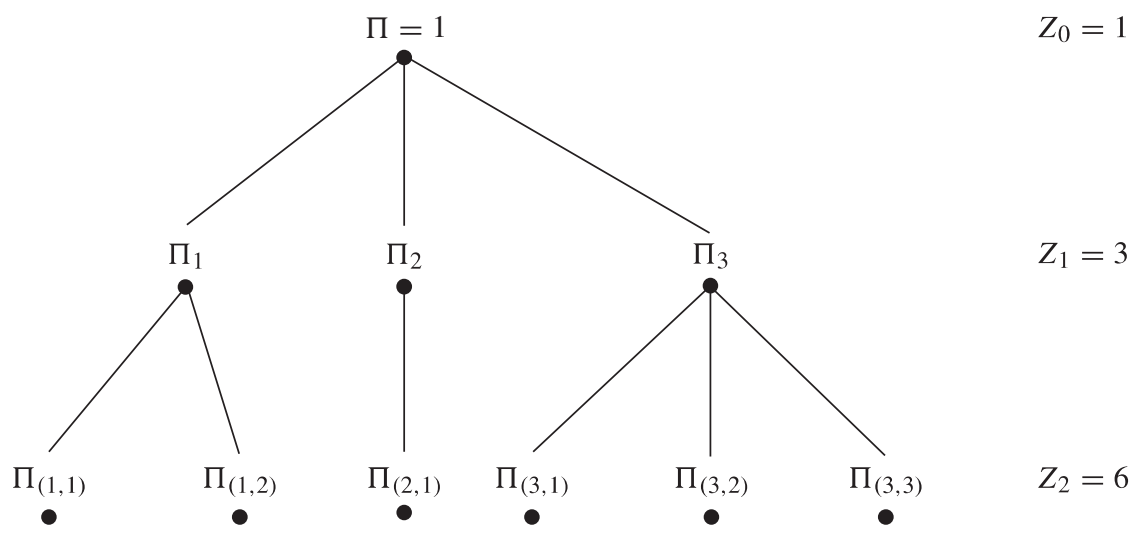

FIGURE 1: Weighted branching tree.

This tree can be obtained from $\mathcal{T}_{Q, C}$ by simply disregarding the values for $Q_{(\cdot)}$ and is denoted by $\mathcal{T}_{C}$.

Studying the tail behavior of the solutions to recursions and fixed-point equations embedded in this weighted branching tree is the objective of this paper.

\section{Implicit renewal theorem on trees}

In this section we present an extension of Goldie's implicit renewal theorem [14] to weighted branching trees. The observation that facilitates this generalization is the following lemma, which shows that a certain measure on a tree is actually a product measure; a similar measure was used in a different context in [9]. Its proof is given in Section 7.1 for completeness. Throughout the paper, we use the standard convention that $0^{\alpha} \log 0=0$ for all $\alpha>0$.

Lemma 3.1. Let $\mathcal{T}_{C}$ be the weighted branching tree defined by the nonnegative vector $\left(N, C_{1}\right.$, $\left.C_{2}, \ldots\right)$, where $N \in \mathbb{N} \cup\{\infty\}$. For any $n \in \mathbb{N}$ and $i \in A_{n}$, let $V_{i}=\log \Pi_{i}$. For $\alpha>0$, define the measure

$$
\mu_{n}(\mathrm{~d} t)=\mathrm{e}^{\alpha t} \mathrm{E}\left[\sum_{i \in A_{n}} \mathbf{1}\left(V_{i} \in \mathrm{d} t\right)\right], \quad n=1,2, \ldots,
$$

and let $\eta(\mathrm{d} t)=\mu_{1}(\mathrm{~d} t)$. Suppose that there exists $j \geq 1$ with $\mathrm{P}\left(N \geq j, C_{j}>0\right)>0$ such that the measure $\mathrm{P}\left(\log C_{j} \in \mathrm{d} u, C_{j}>0, N \geq j\right)$ is nonarithmetic, $0<\mathrm{E}\left[\sum_{i=1}^{N} C_{i}^{\alpha} \log C_{i}\right]<\infty$, and $\mathrm{E}\left[\sum_{i=1}^{N} C_{i}^{\alpha}\right]=1$. Then $\eta(\cdot)$ is a nonarithmetic probability measure on $\mathbb{R}$ that places no mass at $-\infty$ and has mean

$$
\int_{-\infty}^{\infty} u \eta(\mathrm{d} u)=\mathrm{E}\left[\sum_{j=1}^{N} C_{j}^{\alpha} \log C_{j}\right] .
$$

Furthermore, $\mu_{n}(\mathrm{~d} t)=\eta^{* n}(\mathrm{~d} t)$, where $\eta^{* n}$ denotes the $n$th convolution of $\eta$ with itself.

We now present a generalization of Goldie's implicit renewal theorem [14] that will enable the analysis of recursions on weighted branching trees. Note that, except for the independence assumption, the random variable $R$ and the vector $\left(N, C_{1}, C_{2}, \ldots\right)$ are arbitrary, and, therefore, the applicability of this theorem goes beyond the recursions that we study here. Throughout the paper, we use $g(x) \sim f(x)$ as $x \rightarrow \infty$ to denote $\lim _{x \rightarrow \infty} g(x) / f(x)=1$. 
Theorem 3.1. Let $\left(N, C_{1}, C_{2}, \ldots\right)$ be a nonnegative random vector, where $N \in \mathbb{N} \cup\{\infty\}$. Suppose that there exists $j \geq 1$ with $\mathrm{P}\left(N \geq j, C_{j}>0\right)>0$ such that the measure $\mathrm{P}\left(\log C_{j} \in\right.$ $\left.\mathrm{d} u, C_{j}>0, N \geq j\right)$ is nonarithmetic. Assume further that $0<\mathrm{E}\left[\sum_{j=1}^{N} C_{j}^{\alpha} \log C_{j}\right]<\infty$, $\mathrm{E}\left[\sum_{j=1}^{N} C_{j}^{\alpha}\right]=1, \mathrm{E}\left[\sum_{j=1}^{N} C_{j}^{\gamma}\right]<\infty$ for some $0 \leq \gamma<\alpha$, and that $R$ is independent of $\left(N, C_{1}, C_{2}, \ldots\right)$ with $\mathrm{E}\left[R^{\beta}\right]<\infty$ for any $0<\beta<\alpha$. If

$$
\int_{0}^{\infty}\left|\mathrm{P}(R>t)-\mathrm{E}\left[\sum_{j=1}^{N} \mathbf{1}\left(C_{j} R>t\right)\right]\right| t^{\alpha-1} \mathrm{~d} t<\infty
$$

then

$$
\mathrm{P}(R>t) \sim H t^{-\alpha} \quad \text { as } t \rightarrow \infty
$$

where $0 \leq H<\infty$ is given by

$$
H=\frac{1}{\mathrm{E}\left[\sum_{j=1}^{N} C_{j}^{\alpha} \log C_{j}\right]} \int_{0}^{\infty} v^{\alpha-1}\left(\mathrm{P}(R>v)-\mathrm{E}\left[\sum_{j=1}^{N} \mathbf{1}\left(C_{j} R>v\right)\right]\right) \mathrm{d} v .
$$

Remarks. (i) As pointed out in [14], the statement of Theorem 3.1 only has content when $R$ has infinite moment of order $\alpha$, since otherwise the constant $H$ is zero.

(ii) Similarly as in [14], Theorem 3.1 can be generalized to incorporate negative weights $\left\{C_{i}\right\}$ at the expense of additional technical complications. However, when the $\left\{C_{i}\right\} \geq 0$ and $R$ is real valued, we can use exactly the same proof to derive the asymptotics of $\mathrm{P}(-R>t)$; we omit the statement here since our applications do not require it.

(iii) When the $\left\{\log C_{i}\right\}$ are lattice valued, a similar version of Theorem 3.1 can be derived by using the corresponding renewal theorem for lattice random walks.

(iv) It appears, as noted in [14], that some of the early ideas of applying renewal theory to study the power-tail asymptotics of autoregressive processes (perpetuities) is due to Kesten [21] and Grincevičius [15]. The proof given below follows the corresponding proof in [14].

Proof of Theorem 3.1. Let $\mathcal{T}_{C}$ be the weighted branching tree defined by the nonnegative vector $\left(N, C_{1}, C_{2}, \ldots\right)$. For each $i \in A_{n}$ and all $k \leq n$, define $V_{i \mid k}=\log \Pi_{i \mid k}$; note that $\Pi_{\boldsymbol{i} \mid k}$ is independent of $N_{\boldsymbol{i} \mid k}$ but not of $N_{\boldsymbol{i} \mid s}$ for any $0 \leq s \leq k-1$. Also, note that $\boldsymbol{i} \mid n=\boldsymbol{i}$ since $i \in A_{n}$. Let $\mathcal{F}_{k}, k \geq 1$, denote the $\sigma$-algebra generated by $\left\{\left(N_{i}, C_{(i, 1)}, C_{(i, 2)}, \ldots\right): i \in\right.$ $\left.A_{j}, 0 \leq j \leq k-1\right\}$, and let $\mathcal{F}_{0}=\sigma(\varnothing, \Omega), \Pi_{i \mid 0} \equiv 1$. Assume also that $R$ is independent of the entire weighted tree, $\mathcal{T}_{C}$. Then, for any $t \in \mathbb{R}$, we can write $\mathrm{P}\left(R>\mathrm{e}^{t}\right)$ via a telescoping sum as follows (note that all the expectations in (3.2) are finite by Markov's inequality and (3.6) below):

$$
\begin{aligned}
\mathrm{P}\left(R>\mathrm{e}^{t}\right)= & \sum_{k=0}^{n-1}\left(\mathrm{E}\left[\sum_{(i \mid k) \in A_{k}} \mathbf{1}\left(\Pi_{i \mid k} R>\mathrm{e}^{t}\right)\right]-\mathrm{E}\left[\sum_{(i \mid k+1) \in A_{k+1}} \mathbf{1}\left(\Pi_{i \mid k+1} R>\mathrm{e}^{t}\right)\right]\right) \\
& +\mathrm{E}\left[\sum_{(i \mid n) \in A_{n}} \mathbf{1}\left(\Pi_{i \mid n} R>\mathrm{e}^{t}\right)\right] \\
= & \sum_{k=0}^{n-1} \mathrm{E}\left[\sum_{(i \mid k) \in A_{k}}\left(\mathbf{1}\left(\Pi_{i \mid k} R>\mathrm{e}^{t}\right)-\sum_{j=1}^{N_{i \mid k}} \mathbf{1}\left(\Pi_{i \mid k} C_{(\boldsymbol{i} \mid k, j)} R>\mathrm{e}^{t}\right)\right)\right] \\
& +\mathrm{E}\left[\sum_{(i \mid n) \in A_{n}} \mathbf{1}\left(\Pi_{i \mid n} R>\mathrm{e}^{t}\right)\right]
\end{aligned}
$$




$$
\begin{aligned}
= & \sum_{k=0}^{n-1} \mathrm{E}\left[\sum_{(i \mid k) \in A_{k}} \mathrm{E}\left[\mathbf{1}\left(R>\mathrm{e}^{t-V_{i \mid k}}\right)-\sum_{j=1}^{N_{i \mid k}} \mathbf{1}\left(C_{(i \mid k, j)} R>\mathrm{e}^{t-V_{i \mid k}}\right) \mid \mathcal{F}_{k}\right]\right] \\
& +\mathrm{E}\left[\sum_{(i \mid n) \in A_{n}} \mathbf{1}\left(\Pi_{i \mid n} R>\mathrm{e}^{t}\right)\right] .
\end{aligned}
$$

Now, define the measures $\mu_{n}$ according to Lemma 3.1, and let

$$
\begin{gathered}
v_{n}(\mathrm{~d} t)=\sum_{k=0}^{n} \mu_{k}(\mathrm{~d} t), \quad g(t)=\mathrm{e}^{\alpha t}\left(\mathrm{P}\left(R>\mathrm{e}^{t}\right)-\mathrm{E}\left[\sum_{j=1}^{N} \mathbf{1}\left(C_{j} R>\mathrm{e}^{t}\right)\right]\right), \\
r(t)=\mathrm{e}^{\alpha t} \mathrm{P}\left(R>\mathrm{e}^{t}\right), \quad \text { and } \quad \delta_{n}(t)=\mathrm{e}^{\alpha t} \mathrm{E}\left[\sum_{(i \mid n) \in A_{n}} \mathbf{1}\left(\Pi_{i \mid n} R>\mathrm{e}^{t}\right)\right] .
\end{gathered}
$$

Recall that $R$ and $\left(N_{i \mid k}, C_{(i \mid k, 1)}, C_{(i \mid k, 2)}, \ldots\right)$ are independent of $\mathcal{F}_{k}$, from where it follows that

$$
\mathrm{E}\left[\mathbf{1}\left(R>\mathrm{e}^{t-V_{i \mid k}}\right)-\sum_{j=1}^{N_{i \mid k}} \mathbf{1}\left(C_{(\boldsymbol{i} \mid k, j)} R>\mathrm{e}^{t-V_{i \mid k}}\right) \mid \mathcal{F}_{k}\right]=\mathrm{e}^{\alpha\left(V_{i \mid k}-t\right)} g\left(t-V_{i \mid k}\right) .
$$

Then, for any $t \in \mathbb{R}$ and $n \in \mathbb{N}$,

$$
r(t)=\sum_{k=0}^{n-1} \mathrm{E}\left[\sum_{(i \mid k) \in A_{k}} \mathrm{e}^{\alpha V_{i \mid k}} g\left(t-V_{i \mid k}\right)\right]+\delta_{n}(t)=\left(g * v_{n-1}\right)(t)+\delta_{n}(t) .
$$

Next, define the operator $\breve{f}(t)=\int_{-\infty}^{t} \mathrm{e}^{-(t-u)} f(u) \mathrm{d} u$ and note that

$$
\breve{r}(t)=\left(\breve{g} * v_{n-1}\right)(t)+\breve{\delta}_{n}(t) .
$$

Now, we will show that one can let $n \rightarrow \infty$ in the preceding identity. To this end, let $\eta(\mathrm{d} u)=\mu_{1}(\mathrm{~d} u)$, and note that, by Lemma $3.1, \eta(\cdot)$ is a nonarithmetic probability measure on $\mathbb{R}$ that places no mass at $-\infty$ and has mean

$$
\mu:=\int_{-\infty}^{\infty} u \eta(\mathrm{d} u)=\mathrm{E}\left[\sum_{j=1}^{N} C_{j}^{\alpha} \log C_{j}\right]>0 .
$$

Moreover, by Lemma 3.1,

$$
v(\mathrm{~d} t):=\sum_{k=0}^{\infty} \mathrm{e}^{\alpha t} \mathrm{E}\left[\sum_{(i \mid k) \in A_{k}} \mathbf{1}\left(V_{\boldsymbol{i} \mid k} \in \mathrm{d} t\right)\right]=\sum_{k=0}^{\infty} \eta^{* k}(\mathrm{~d} t)
$$

is its renewal measure. Since $\mu \neq 0$, then $(|f| * v)(t)<\infty$ for all $t$ whenever $f$ is directly Riemann integrable. By (3.1) we know that $g \in L^{1}$, so, by Lemma 9.1 of [14], $\breve{g}$ is directly Riemann integrable, resulting in $(|\breve{g}| * v)(t)<\infty$ for all $t$. Thus,

$$
(|\breve{g}| * v)(t)=\mathrm{E}\left[\sum_{k=0}^{\infty} \sum_{(i \mid k) \in A_{k}} \mathrm{e}^{\alpha V_{i \mid k}}\left|\breve{g}\left(t-V_{i \mid k}\right)\right|\right]<\infty
$$




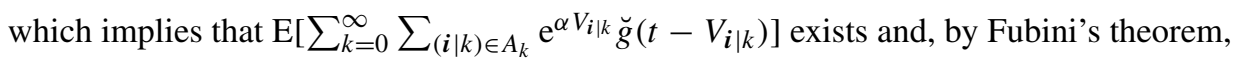

$$
\begin{aligned}
(\breve{g} * v)(t) & =\mathrm{E}\left[\sum_{k=0}^{\infty} \sum_{(i \mid k) \in A_{k}} \mathrm{e}^{\left.\alpha V_{i \mid k} \breve{g}\left(t-V_{i \mid k}\right)\right]}\right. \\
& =\sum_{k=0}^{\infty} \mathrm{E}\left[\sum_{(i \mid k) \in A_{k}} \mathrm{e}^{\alpha V_{i \mid k}} \breve{g}\left(t-V_{i \mid k}\right)\right] \\
& =\lim _{n \rightarrow \infty}\left(\breve{g} * v_{n}\right)(t) .
\end{aligned}
$$

To see that $\breve{\delta}_{n}(t) \rightarrow 0$ as $n \rightarrow \infty$ for all fixed $t$, note that, from the assumptions that $0<\mathrm{E}\left[\sum_{j=1}^{N} C_{j}^{\alpha} \log C_{j}\right]<\infty, \mathrm{E}\left[\sum_{j=1}^{N} C_{j}^{\alpha}\right]=1$, and $\mathrm{E}\left[\sum_{j=1}^{N} C_{j}^{\gamma}\right]<\infty$ for some $0 \leq$ $\gamma<\alpha$, there exists $0<\beta<\alpha$ such that $\mathrm{E}\left[\sum_{j=1}^{N} C_{j}^{\beta}\right]<1$ (by convexity). Then, for such $\beta$,

$$
\begin{aligned}
\breve{\delta}_{n}(t) & =\int_{-\infty}^{t} \mathrm{e}^{-(t-u)} \mathrm{e}^{\alpha u} \mathrm{E}\left[\sum_{(i \mid n) \in A_{n}} \mathbf{1}\left(\Pi_{i \mid n} R>\mathrm{e}^{u}\right)\right] \mathrm{d} u \\
& \leq \mathrm{e}^{(\alpha-\beta) t} \mathrm{E}\left[\sum_{(i \mid n) \in A_{n}} \int_{-\infty}^{t} \mathrm{e}^{\beta u} \mathbf{1}\left(\Pi_{i \mid n} R>\mathrm{e}^{u}\right) \mathrm{d} u\right] \\
& =\mathrm{e}^{(\alpha-\beta) t} \mathrm{E}\left[\sum_{(i \mid n) \in A_{n}} \int_{-\infty}^{\min \left\{t, \log \left(\Pi_{i \mid n} R\right)\right\}} \mathrm{e}^{\beta u} \mathrm{~d} u\right] \\
& \leq \frac{\mathrm{e}^{(\alpha-\beta) t}}{\beta} \mathrm{E}\left[\sum_{(i \mid n) \in A_{n}}\left(\Pi_{i \mid n} R\right)^{\beta}\right] .
\end{aligned}
$$

It remains to show that the expectation in (3.5) converges to 0 as $n \rightarrow \infty$. First note that, from the independence of $R$ and $\mathcal{T}_{C}$,

$$
\mathrm{E}\left[\sum_{(i \mid n) \in A_{n}}\left(\Pi_{i \mid n} R\right)^{\beta}\right]=\mathrm{E}\left[R^{\beta}\right] \mathrm{E}\left[\sum_{(i \mid n) \in A_{n}}\left(\Pi_{i \mid n}\right)^{\beta}\right],
$$

where $\mathrm{E}\left[R^{\beta}\right]<\infty$ for $0<\beta<\alpha$. For the expectation involving $\Pi_{i \mid n}$, condition on $\mathcal{F}_{n-1}$ and use the independence of $\left(N_{\boldsymbol{i} \mid n-1}, C_{(\boldsymbol{i} \mid n-1,1)}, C_{(\boldsymbol{i} \mid n-1,2)}, \ldots\right)$ from $\mathcal{F}_{n-1}$ as follows:

$$
\begin{aligned}
\mathrm{E}\left[\sum_{(\boldsymbol{i} \mid n) \in A_{n}}\left(\Pi_{i \mid n}\right)^{\beta}\right] & =\mathrm{E}\left[\sum_{(i \mid n-1) \in A_{n-1}} \mathrm{E}\left[\sum_{j=1}^{N_{i \mid n-1}}\left(\Pi_{i \mid n-1}\right)^{\beta} C_{(i \mid n-1, j)}^{\beta} \mid \mathcal{F}_{n-1}\right]\right] \\
& =\mathrm{E}\left[\sum_{(i \mid n-1) \in A_{n-1}}\left(\Pi_{i \mid n-1}\right)^{\beta} \mathrm{E}\left[\sum_{j=1}^{N_{i \mid n-1}} C_{(i \mid n-1, j)}^{\beta} \mid \mathcal{F}_{n-1}\right]\right] \\
& =\mathrm{E}\left[\sum_{j=1}^{N} C_{j}^{\beta}\right] \mathrm{E}\left[\sum_{(i \mid n-1) \in A_{n-1}}\left(\Pi_{i \mid n-1}\right)^{\beta}\right] \\
& \left.=\left(\mathrm{E}\left[\sum_{j=1}^{N} C_{j}^{\beta}\right]\right)^{n} \text { (iterating } n-1 \text { times }\right) .
\end{aligned}
$$


Since $\mathrm{E}\left[\sum_{j=1}^{N} C_{j}^{\beta}\right]<1$, then the above converges to 0 as $n \rightarrow \infty$. Hence, the preceding arguments allow us to pass $n \rightarrow \infty$ in (3.3), and obtain

$$
\breve{r}(t)=(\breve{g} * v)(t) .
$$

Now, by the key renewal theorem for two-sided random walks (see Theorem 4.2 of [7]),

$$
\mathrm{e}^{-t} \int_{0}^{\mathrm{e}^{t}} v^{\alpha} \mathrm{P}(R>v) \mathrm{d} v=\breve{r}(t) \rightarrow \frac{1}{\mu} \int_{-\infty}^{\infty} \breve{g}(u) \mathrm{d} u=: H \quad \text { as } t \rightarrow \infty .
$$

Clearly, $H \geq 0$ since the left-hand side of the preceding equation is positive, and, thus, by Lemma 9.3 of [14],

$$
\mathrm{P}(R>t) \sim H t^{-\alpha} \text { as } t \rightarrow \infty
$$

Finally,

$$
\begin{aligned}
H & =\frac{1}{\mu} \int_{-\infty}^{\infty} \int_{-\infty}^{u} \mathrm{e}^{-(u-t)} g(t) \mathrm{d} t \mathrm{~d} u \\
& =\frac{1}{\mu} \int_{-\infty}^{\infty} \mathrm{e}^{t} g(t) \int_{t}^{\infty} \mathrm{e}^{-u} \mathrm{~d} u \mathrm{~d} t \\
& =\frac{1}{\mu} \int_{-\infty}^{\infty} g(t) \mathrm{d} t \\
& =\frac{1}{\mu} \int_{-\infty}^{\infty} \mathrm{e}^{\alpha t}\left(\mathrm{P}\left(R>\mathrm{e}^{t}\right)-\mathrm{E}\left[\sum_{j=1}^{N} \mathbf{1}\left(C_{j} R>\mathrm{e}^{t}\right)\right]\right) \mathrm{d} t \\
& =\frac{1}{\mu} \int_{0}^{\infty} v^{\alpha-1}\left(\mathrm{P}(R>v)-\mathrm{E}\left[\sum_{j=1}^{N} \mathbf{1}\left(C_{j} R>v\right)\right]\right) \mathrm{d} v .
\end{aligned}
$$

\section{The linear recursion: $R=\sum_{i=1}^{N} C_{i} R_{i}+Q$}

Motivated by the information ranking problem on the Internet, e.g. Google's PageRank algorithm [19], [27], in this section we apply the implicit renewal theory for trees developed in the previous section to the linear recursion

$$
R \stackrel{\mathrm{D}}{=} \sum_{i=1}^{N} C_{i} R_{i}+Q
$$

where $\left(Q, N, C_{1}, C_{2}, \ldots\right)$ is a nonnegative random vector with $N \in \mathbb{N} \cup\{\infty\}, \mathrm{P}(Q>0)>0$, and $\left\{R_{i}\right\}_{i \in \mathbb{N}}$ is a sequence of i.i.d. random variables independent of $\left(Q, N, C_{1}, C_{2}, \ldots\right)$ with the same distribution as $R$. Note that the power tail of $R$ in the critical homogeneous case $(Q \equiv 0)$ was previously studied in [17] and [23]. In Section 4.3 we will give an alternative derivation of those results using our method and we will provide pointers to the appropriate literature.

As for the nonhomogeneous case, the first result we need to establish is the existence and finiteness of a solution to (4.1). For the purpose of existence, we will provide an explicit construction of the solution $R$ to (4.1) on a tree. Note that such constructed $R$ will be the main object of study in this section.

Recall that throughout the paper the convention is to denote the random vector associated to the root node $\varnothing$ by $\left(Q, N, C_{1}, C_{2}, \ldots\right) \equiv\left(Q_{\varnothing}, N_{\varnothing}, C_{(\varnothing, 1)}, C_{(\varnothing, 2)}, \ldots\right)$. 
We now define the process

$$
W_{0}=Q, \quad W_{n}=\sum_{i \in A_{n}} Q_{i} \Pi_{i}, \quad n \geq 1,
$$

on the weighted branching tree $\mathcal{T}_{Q, C}$, as constructed in Section 2. Define the process $\left\{R^{(n)}\right\}_{n \geq 0}$ according to

$$
R^{(n)}=\sum_{k=0}^{n} W_{k}, \quad n \geq 0,
$$

that is, $R^{(n)}$ is the sum of the weights of all the nodes on the tree up to the $n$th generation. It is not hard to see that $R^{(n)}$ satisfies the recursion

$$
R^{(n)}=\sum_{j=1}^{N_{\varnothing}} C_{(\varnothing, j)} R_{j}^{(n-1)}+Q_{\varnothing}=\sum_{j=1}^{N} C_{j} R_{j}^{(n-1)}+Q, \quad n \geq 1,
$$

where $\left\{R_{j}^{(n-1)}\right\}$ are independent copies of $R^{(n-1)}$ corresponding to the tree starting with individual $j$ in the first generation and ending on the $n$th generation; note that $R_{j}^{(0)}=Q_{j}$. Similarly, since the tree structure repeats itself after the first generation, $W_{n}$ satisfies

$$
\begin{aligned}
W_{n} & =\sum_{i \in A_{n}} Q_{i} \Pi_{i} \\
& =\sum_{k=1}^{N_{\varnothing}} C_{(\varnothing, k)} \sum_{\left(k, \ldots, i_{n}\right) \in A_{n}} Q_{\left(k, \ldots, i_{n}\right)} \prod_{j=2}^{n} C_{\left(k, \ldots, i_{j}\right)} \\
& \stackrel{\mathrm{D}}{=} \sum_{k=1}^{N} C_{k} W_{(n-1), k},
\end{aligned}
$$

where $\left\{W_{(n-1), k}\right\}$ is a sequence of i.i.d. random variables independent of $\left(N, C_{1}, C_{2}, \ldots\right)$ and having the same distribution as $W_{n-1}$.

Next, define the random variable $R$ according to

$$
R:=\lim _{n \rightarrow \infty} R^{(n)}=\sum_{k=0}^{\infty} W_{k},
$$

where the limit is properly defined by (4.3) and monotonicity. Hence, it is easy to verify, by applying monotone convergence in (4.4), that $R$ must solve

$$
R=\sum_{j=1}^{N_{\varnothing}} C_{(\varnothing, j)} R_{j}^{(\infty)}+Q_{\varnothing}=\sum_{j=1}^{N} C_{j} R_{j}^{(\infty)}+Q
$$

where $\left\{R_{j}^{(\infty)}\right\}_{j \in \mathbb{N}}$ are i.i.d., have the same distribution as $R$, and are independent of $\left(Q, N, C_{1}\right.$, $\left.C_{2}, \ldots\right)$.

The derivation provided above implies in particular the existence of a solution in distribution to (4.1). Moreover, under additional technical conditions, $R$ is the unique solution under iterations, as we will define and show in the following section. The constructed $R$, as defined in (4.6), is the main object of study in the remainder of this section. 


\subsection{Moments of $W_{n}$ and $R$}

In this section we derive estimates for the moments of $W_{n}$ and $R$. We start by stating a lemma about the moments of a sum of random variables. The proofs of Lemmas 4.1, 4.2, and 4.3 are given in Section 7.2.

Lemma 4.1. For any $k \in \mathbb{N} \cup\{\infty\}$, let $\left\{C_{i}\right\}_{i=1}^{k}$ be a sequence of nonnegative random variables and let $\left\{Y_{i}\right\}_{i=1}^{k}$ be a sequence of nonnegative i.i.d. random variables, independent of the $\left\{C_{i}\right\}$, with the same distribution as $Y$. For $\beta>1$, set $p=\lceil\beta\rceil \in\{2,3,4, \ldots\}$, and if $k=\infty$, assume that $\sum_{i=1}^{\infty} C_{i} Y_{i}<\infty$ almost surely (a.s.). Then,

$$
\mathrm{E}\left[\left(\sum_{i=1}^{k} C_{i} Y_{i}\right)^{\beta}-\sum_{i=1}^{k}\left(C_{i} Y_{i}\right)^{\beta}\right] \leq\left(\mathrm{E}\left[Y^{p-1}\right]\right)^{\beta /(p-1)} \mathrm{E}\left[\left(\sum_{i=1}^{k} C_{i}\right)^{\beta}\right] .
$$

Remark. Note that Lemma 4.1 does not exclude the case when $\mathrm{E}\left[\left(\sum_{i=1}^{k} C_{i} Y_{i}\right)^{\beta}\right]=\infty$ but $\mathrm{E}\left[\left(\sum_{i=1}^{k} C_{i} Y_{i}\right)^{\beta}-\sum_{i=1}^{k}\left(C_{i} Y_{i}\right)^{\beta}\right]<\infty$.

We now give estimates for the $\beta$-moments of $W_{n}$ for $\beta \in(0,1]$ and $\beta>1$ in Lemmas 4.2 and 4.3 , respectively. Throughout the rest of the paper, we define $\rho_{\beta}=\mathrm{E}\left[\sum_{i=1}^{N} C_{i}^{\beta}\right]$ for any $\beta>0$, and $\rho \equiv \rho_{1}$.

Lemma 4.2. For $0<\beta \leq 1$ and all $n \geq 0$,

$$
\mathrm{E}\left[W_{n}^{\beta}\right] \leq \mathrm{E}\left[Q^{\beta}\right] \rho_{\beta}^{n}
$$

Lemma 4.3. For $\beta>1$, suppose that $\mathrm{E}\left[Q^{\beta}\right]<\infty, \mathrm{E}\left[\left(\sum_{i=1}^{N} C_{i}\right)^{\beta}\right]<\infty$, and $\rho \vee \rho_{\beta}<1$. Then, there exists a constant $K_{\beta}>0$ such that, for all $n \geq 0$,

$$
\mathrm{E}\left[W_{n}^{\beta}\right] \leq K_{\beta}\left(\rho \vee \rho_{\beta}\right)^{n} .
$$

Now we are ready to establish the finiteness of moments of the solution $R$ given by (4.6). The proof of this lemma uses well-known contraction arguments, but for completeness we provide the details below.

Lemma 4.4. Assume that $\mathrm{E}\left[Q^{\beta}\right]<\infty$ for some $\beta>0$. In addition, suppose that either

(i) $\rho_{\beta}<1$ if $0<\beta<1$, or

(ii) $\left(\rho \vee \rho_{\beta}\right)<1$ and $\mathrm{E}\left[\left(\sum_{i=1}^{N} C_{i}\right)^{\beta}\right]<\infty$ if $\beta \geq 1$.

Then, $\mathrm{E}\left[R^{\gamma}\right]<\infty$ for all $0<\gamma \leq \beta$, and, in particular, $R<\infty$ a.s. Moreover, if $\beta \geq 1$, $R^{(n)} \stackrel{L_{\beta}}{\longrightarrow} R$, where $L_{\beta}$ stands for convergence in the $\left(\mathrm{E}|\cdot|^{\beta}\right)^{1 / \beta}$ norm.

Remark. It is interesting to observe that, for $\beta>1$, the conditions $\mathrm{E}\left[\left(\sum_{i=1}^{N} C_{i}\right)^{\beta}\right]<\infty$ and $\rho_{\beta}<1$ are consistent with Theorem 3.1 of [2], Proposition 4 of [17], and Theorem 2.1 of [23], which give the conditions for the finiteness of the $\beta$-moment of the solution to the related critical $\left(\rho_{1}=1\right)$ homogeneous $(Q \equiv 0)$ equation.

Proof of Lemma 4.4. Let

$$
\eta= \begin{cases}\rho_{\beta} & \text { if } \beta<1 \\ \rho \vee \rho_{\beta} & \text { if } \beta \geq 1\end{cases}
$$

Then, by Lemmas 4.2 and 4.3,

$$
\mathrm{E}\left[W_{n}^{\beta}\right] \leq K \eta^{n} \quad \text { for some } K>0 .
$$


Suppose that $\beta \geq 1$. Then, by monotone convergence and Minkowski's inequality,

$$
\begin{aligned}
\mathrm{E}\left[R^{\beta}\right] & =\mathrm{E}\left[\lim _{n \rightarrow \infty}\left(\sum_{k=0}^{n} W_{k}\right)^{\beta}\right] \\
& =\lim _{n \rightarrow \infty} \mathrm{E}\left[\left(\sum_{k=0}^{n} W_{k}\right)^{\beta}\right] \\
& \leq \lim _{n \rightarrow \infty}\left(\sum_{k=0}^{n}\left(\mathrm{E}\left[W_{k}^{\beta}\right]\right)^{1 / \beta}\right)^{\beta} \\
& \leq K\left(\sum_{k=0}^{\infty} \eta^{k / \beta}\right)^{\beta} \\
& <\infty .
\end{aligned}
$$

This implies that $R<\infty$ a.s. When $0<\beta \leq 1$, use the inequality $\left(\sum_{k=0}^{n} y_{k}\right)^{\beta} \leq \sum_{k=0}^{n} y_{k}^{\beta}$ for any $y_{i} \geq 0$ instead of Minkowski's inequality. Furthermore, for any $0<\gamma \leq \beta$,

$$
\mathrm{E}\left[R^{\gamma}\right]=\mathrm{E}\left[\left(R^{\beta}\right)^{\gamma / \beta}\right] \leq\left(\mathrm{E}\left[R^{\beta}\right]\right)^{\gamma / \beta}<\infty .
$$

That $R^{(n)} \stackrel{L_{\beta}}{\longrightarrow} R$ whenever $\beta \geq 1$ follows from noting that

$$
\mathrm{E}\left[\left|R^{(n)}-R\right|^{\beta}\right]=\mathrm{E}\left[\left(\sum_{k=n+1}^{\infty} W_{k}\right)^{\beta}\right]
$$

and applying the same arguments used above to obtain the bound

$$
\mathrm{E}\left[\left|R^{(n)}-R\right|^{\beta}\right] \leq \frac{K \eta^{n+1}}{\left(1-\eta^{1 / \beta}\right)^{\beta}} .
$$

This completes the proof.

Next, we show that, under some technical conditions, the iteration of recursion (4.1) results in a process that converges in distribution to $R$ for any initial condition $R_{0}^{*}$. To this end, consider a weighted branching tree $\mathcal{T}_{Q, C}$, as defined in Section 2 . Now, define

$$
R_{n}^{*}:=R^{(n-1)}+W_{n}\left(R_{0}^{*}\right), \quad n \geq 1,
$$

where $R^{(n-1)}$ is given by (4.3),

$$
W_{n}\left(R_{0}^{*}\right)=\sum_{i \in A_{n}} R_{0, i}^{*} \Pi_{i}
$$

and $\left\{R_{0, i}^{*}\right\}_{i \in U}$ are i.i.d. copies of an initial value $R_{0}^{*}$, independent of the entire weighted tree $\mathcal{T}_{Q, C}$. It follows from (4.4) and (4.7) that, for $n \geq 0$,

$R_{n+1}^{*}=\sum_{j=1}^{N} C_{j} R_{j}^{(n-1)}+Q+W_{n+1}\left(R_{0}^{*}\right)=\sum_{j=1}^{N} C_{j}\left(R_{j}^{(n-1)}+\sum_{i \in A_{n, j}} R_{0, i}^{*} \prod_{k=2}^{n} C_{\left(j, \ldots, i_{k}\right)}\right)+Q$,

where $\left\{R_{j}^{(n-1)}\right\}$ are independent copies of $R^{(n-1)}$ corresponding to the tree starting with individual $j$ in the first generation and ending on the $n$th generation, and $A_{n, j}$ is the set of all nodes in the $(n+1)$ th generation that are descendants of individual $j$ in the first generation. 
It follows that

$$
R_{n+1}^{*}=\sum_{j=1}^{N} C_{j} R_{n, j}^{*}+Q,
$$

where $\left\{R_{n, j}^{*}\right\}$ are the expressions inside the parenthesis in (4.8). Clearly, $\left\{R_{n, j}^{*}\right\}$ are i.i.d. copies of $R_{n}^{*}$; thus, we show that $R_{n}^{*}$ is equal in distribution to the process derived by iterating (4.1) with an initial condition $R_{0}^{*}$. The following lemma shows that $R_{n}^{*} \Rightarrow R$ for any initial condition $R_{0}^{*}$ satisfying a moment assumption, where ' $\Rightarrow$ ' denotes convergence in distribution.

Lemma 4.5. For any initial condition $R_{0}^{*} \geq 0$, if $\mathrm{E}\left[Q^{\beta}\right], \mathrm{E}\left[\left(R_{0}^{*}\right)^{\beta}\right]<\infty$, and $\rho_{\beta}=$ $\mathrm{E}\left[\sum_{i=1}^{N} C_{i}^{\beta}\right]<1$ for some $0<\beta \leq 1$, then

$$
R_{n}^{*} \Rightarrow R
$$

with $\mathrm{E}\left[R^{\beta}\right]<\infty$. Furthermore, under these assumptions, the distribution of $R$ is the unique solution with finite $\beta$-moment to recursion (4.1).

Proof. Since $R^{(n)} \rightarrow R$ a.s., the result will follow from Slutsky's theorem (see Theorem 25.4 of $\left[10\right.$, p. 332]) once we show that $W_{n}\left(R_{0}^{*}\right) \Rightarrow 0$. To this end, note that $W_{n}\left(R_{0}^{*}\right)$, as defined by (4.7), is the same as $W_{n}$ if we substitute the $Q_{i}$ by the $R_{0, i}^{*}$. Then, for every $\varepsilon>0$, we have

$$
\mathrm{P}\left(W_{n}\left(R_{0}^{*}\right)>\varepsilon\right) \leq \varepsilon^{-\beta} \mathrm{E}\left[W_{n}\left(R_{0}^{*}\right)^{\beta}\right] \leq \varepsilon^{-\beta} \rho_{\beta}^{n} \mathrm{E}\left[\left(R_{0}^{*}\right)^{\beta}\right] \quad \text { (by Lemma 4.2). }
$$

Since, by assumption, the right-hand side converges to 0 as $n \rightarrow \infty$, then $R_{n}^{*} \Rightarrow R$. Furthermore, $\mathrm{E}\left[R^{\beta}\right]<\infty$ by Lemma 4.4. Clearly, under the assumptions, the distribution of $R$ represents the unique solution to (4.1), since any other possible solution with finite $\beta$-moment would have to converge to the same limit.

Remarks. (i) Note that, when $\mathrm{E}[N]<1$, the branching tree is a.s. finite and no conditions on the $\left\{C_{i}\right\}$ are necessary for $R<\infty$ a.s. This corresponds to the second condition in Theorem 1 of [11].

(ii) In view of the same theorem from [11], one could possibly establish the convergence of $R_{n}^{*} \Rightarrow R<\infty$ under milder conditions. However, since in this paper we only study the power tails of $R$, the assumptions of Lemma 4.5 are not restrictive.

(iii) Note that if $\mathrm{E}\left[\sum_{i=1}^{N} C_{i}^{\alpha}\right]=1$ with $\alpha \in(0,1]$ then there might not be a $0<\beta<\alpha$ for which $\mathrm{E}\left[\sum_{i=1}^{N} C_{i}^{\beta}\right]<1$, e.g. the case of deterministic $C_{i}$ s that was studied in [25].

\subsection{Main result}

We now characterize the tail behavior of the distribution of the solution $R$ to the nonhomogeneous equation (4.1), as defined by (4.6).

Theorem 4.1. Let $\left(Q, N, C_{1}, C_{2}, \ldots\right)$ be a nonnegative random vector, with $N \in \mathbb{N} \cup\{\infty\}$ and $\mathrm{P}(Q>0)>0$, and let $R$ be the solution to (4.1) given by (4.6). Suppose that there exists $j \geq 1$ with $\mathrm{P}\left(N \geq j, C_{j}>0\right)>0$ such that the measure $\mathrm{P}\left(\log C_{j} \in \mathrm{d} u, C_{j}>0, N \geq j\right)$ is nonarithmetic, and that, for some $\alpha>0, \mathrm{E}\left[Q^{\alpha}\right]<\infty, 0<\mathrm{E}\left[\sum_{i=1}^{N} C_{i}^{\alpha} \log C_{i}\right]<\infty$, and $\mathrm{E}\left[\sum_{i=1}^{N} C_{i}^{\alpha}\right]=1$. In addition, assume that

(a) $\mathrm{E}\left[\sum_{i=1}^{N} C_{i}\right]<1$ and $\mathrm{E}\left[\left(\sum_{i=1}^{N} C_{i}\right)^{\alpha}\right]<\infty$ if $\alpha>1$, or

(b) $\mathrm{E}\left[\left(\sum_{i=1}^{N} C_{i}^{\alpha /(1+\varepsilon)}\right)^{1+\varepsilon}\right]<\infty$ for some $0<\varepsilon<1$ if $0<\alpha \leq 1$. 
Then,

$$
\mathrm{P}(R>t) \sim H t^{-\alpha} \text { as } t \rightarrow \infty,
$$

where $0 \leq H<\infty$ is given by

$$
\begin{aligned}
H & =\frac{1}{\mathrm{E}\left[\sum_{i=1}^{N} C_{i}^{\alpha} \log C_{i}\right]} \int_{0}^{\infty} v^{\alpha-1}\left(\mathrm{P}(R>v)-\mathrm{E}\left[\sum_{i=1}^{N} \mathbf{1}\left(C_{i} R>v\right)\right]\right) \mathrm{d} v \\
& =\frac{\mathrm{E}\left[\left(\sum_{i=1}^{N} C_{i} R_{i}+Q\right)^{\alpha}-\sum_{i=1}^{N}\left(C_{i} R_{i}\right)^{\alpha}\right]}{\alpha \mathrm{E}\left[\sum_{i=1}^{N} C_{i}^{\alpha} \log C_{i}\right]} .
\end{aligned}
$$

Remarks. (i) The nonhomogeneous equation has been previously studied for the special case when $Q$ and the $\left\{C_{i}\right\}$ are deterministic constants. In particular, Theorem 5 of [25] analyzes the solutions to (4.1) when $Q$ and the $\left\{C_{i}\right\}$ are nonnegative deterministic constants, which, when $\sum_{i=1}^{N} C_{i}^{\alpha}=1, \alpha>0$, implies that $C_{i} \leq 1$ for all $i$ and $\sum_{i} C_{i}^{\alpha} \log C_{i} \leq 0$, falling outside the scope of this paper. The solutions to (4.1) for the case when $Q$ and the $C_{i}$ s are real-valued deterministic constants were analyzed in [4]. For the recent work (published on arXiv after the first draft of this paper) that characterizes all the solutions to (4.1) for $Q$ and $\left\{C_{i}\right\}$ random, see [3].

(ii) When $\alpha>1$, the condition $\mathrm{E}\left[\left(\sum_{i=1}^{N} C_{i}\right)^{\alpha}\right]<\infty$ is needed to ensure that the tail of $R$ is not dominated by $N$. In particular, if the $\left\{C_{i}\right\}$ are i.i.d. and independent of $N$, the condition reduces to $\mathrm{E}\left[N^{\alpha}\right]<\infty$ since $\mathrm{E}\left[C^{\alpha}\right]<\infty$ is implied by the other conditions; see Theorems 4.2 and 5.4 of [19]. Furthermore, when $0<\alpha \leq 1$, the condition $\mathrm{E}\left[\left(\sum_{i=1}^{N} C_{i}\right)^{\alpha}\right]<\infty$ is redundant since $\mathrm{E}\left[\left(\sum_{i=1}^{N} C_{i}\right)^{\alpha}\right] \leq \mathrm{E}\left[\sum_{i=1}^{N} C_{i}^{\alpha}\right]=1$, and the additional condition $\mathrm{E}\left[\left(\sum_{i=1}^{N} C_{i}^{\alpha /(1+\varepsilon)}\right)^{1+\varepsilon}\right]<$ $\infty$ is needed. When the $\left\{C_{i}\right\}$ are i.i.d. and independent of $N$, the latter condition reduces to $\mathrm{E}\left[N^{1+\varepsilon}\right]<\infty$ (given the other assumptions), which is consistent with Theorem 4.2 of [19].

(iii) Note that the second expression for $H$ is more suitable for actually computing it, especially in the case of $\alpha$ being an integer, as will be stated in the forthcoming Corollary 4.1. When $\alpha>1$ is not an integer, we can derive an explicit upper bound on $H$ by using Lemma 4.6 below. Regarding the lower bound, the elementary inequality $\left(\sum_{i=1}^{k} x_{i}\right)^{\alpha} \geq \sum_{i=1}^{k} x_{i}^{\alpha}$ for $\alpha \geq 1$ and $x_{i} \geq 0$ yields

$$
H \geq \frac{\mathrm{E}\left[Q^{\alpha}\right]}{\alpha \mathrm{E}\left[\sum_{i=1}^{N} C_{i}^{\alpha} \log C_{i}\right]}>0 .
$$

Similarly, for $0<\alpha<1$, using the corresponding inequality $\left(\sum_{i=1}^{k} x_{i}\right)^{\alpha} \leq \sum_{i=1}^{k} x_{i}^{\alpha}$ for $0<\alpha \leq 1$ and $x_{i} \geq 0$, we obtain $H \leq \mathrm{E}\left[Q^{\alpha}\right] /\left(\alpha \mathrm{E}\left[\sum_{i=1}^{N} C_{i}^{\alpha} \log C_{i}\right]\right)$.

(iv) Let us also observe that the solution $R$, given by (4.6), to (4.1) may be a constant (nonpower law) $R=r>0$ when $\mathrm{P}\left(r=Q+r \sum_{i=1}^{N} C_{i}\right)=1$. However, similarly as in (i), such a solution is excluded from the theorem since $\mathrm{P}\left(r=Q+r \sum_{i=1}^{N} C_{i}\right)=1$ implies that $\mathrm{E}\left[\sum_{i} C_{i}^{\alpha} \log C_{i}\right] \leq 0, \alpha>0$.

Before proceeding with the proof of Theorem 4.1, we need the following two technical results; their proofs are given in Section 7.3. Lemma 4.6 below will also be used in subsequent sections for other recursions. With some abuse of notation, throughout the paper, we will use $\max _{1 \leq i \leq N} x_{i}$ to denote $\sup _{1 \leq i<N+1} x_{i}$ in the case $N=\infty$. 
Lemma 4.6. Suppose that $\left(N, C_{1}, C_{2}, \ldots\right)$ is a nonnegative random vector with $N \in \mathbb{N} \cup\{\infty\}$, and let $\left\{R_{i}\right\}_{i \in \mathbb{N}}$ be a sequence of i.i.d. nonnegative random variables independent of $\left(N, C_{1}\right.$, $\left.C_{2}, \ldots\right)$ with the same distribution as $R$. For $\alpha>0$, suppose that $\sum_{i=1}^{N}\left(C_{i} R_{i}\right)^{\alpha}<\infty$ a.s. and $\mathrm{E}\left[R^{\beta}\right]<\infty$ for any $0<\beta<\alpha$. Furthermore, assume that $\mathrm{E}\left[\left(\sum_{i=1}^{N} C_{i}^{\alpha /(1+\varepsilon)}\right)^{1+\varepsilon}\right]<\infty$ for some $0<\varepsilon<1$. Then

$$
\begin{aligned}
0 & \leq \int_{0}^{\infty}\left(\mathrm{E}\left[\sum_{i=1}^{N} \mathbf{1}\left(C_{i} R_{i}>t\right)\right]-\mathrm{P}\left(\max _{1 \leq i \leq N} C_{i} R_{i}>t\right)\right) t^{\alpha-1} \mathrm{~d} t \\
& =\frac{1}{\alpha} \mathrm{E}\left[\sum_{i=1}^{N}\left(C_{i} R_{i}\right)^{\alpha}-\left(\max _{1 \leq i \leq N} C_{i} R_{i}\right)^{\alpha}\right] \\
& <\infty
\end{aligned}
$$

Lemma 4.7. Let $\left(Q, N, C_{1}, C_{2}, \ldots\right)$ be a nonnegative vector with $N \in \mathbb{N} \cup\{\infty\}$, and let $\left\{R_{i}\right\}$ be a sequence of i.i.d. random variables, independent of $\left(Q, N, C_{1}, C_{2}, \ldots\right)$. Suppose that, for some $\alpha>1$, we have $\mathrm{E}\left[Q^{\alpha}\right]<\infty, \mathrm{E}\left[\left(\sum_{i=1}^{N} C_{i}\right)^{\alpha}\right]<\infty, \mathrm{E}\left[R^{\beta}\right]<\infty$ for any $0<\beta<\alpha$, and $\sum_{i=1}^{N} C_{i} R_{i}<\infty$ a.s. Then

$$
\mathrm{E}\left[\left(\sum_{i=1}^{N} C_{i} R_{i}+Q\right)^{\alpha}-\sum_{i=1}^{N}\left(C_{i} R_{i}\right)^{\alpha}\right]<\infty
$$

Proof of Theorem 4.1. By Lemma 4.4, we know that $\mathrm{E}\left[R^{\beta}\right]<\infty$ for any $0<\beta<\alpha$. To verify that $\mathrm{E}\left[\sum_{i=1}^{N} C_{i}^{\gamma}\right]<\infty$ for some $0 \leq \gamma<\alpha$, note that if $\alpha>1$, we have, by the assumptions of the theorem and Jensen's inequality,

$$
\mathrm{E}\left[\sum_{i=1}^{N} C_{i}^{\gamma}\right] \leq \mathrm{E}\left[\left(\sum_{i=1}^{N} C_{i}\right)^{\gamma}\right] \leq\left(\mathrm{E}\left[\left(\sum_{i=1}^{N} C_{i}\right)^{\alpha}\right]\right)^{\gamma / \alpha}<\infty
$$

for any $1 \leq \gamma<\alpha$. If $0<\alpha \leq 1$ then, for $\gamma=\alpha(1+\varepsilon / 2) /(1+\varepsilon)<\alpha$, we have

$$
\mathrm{E}\left[\sum_{i=1}^{N} C_{i}^{\gamma}\right] \leq \mathrm{E}\left[\left(\sum_{i=1}^{N} C_{i}^{\alpha /(1+\varepsilon)}\right)^{1+\varepsilon / 2}\right] \leq\left(\mathrm{E}\left[\left(\sum_{i=1}^{N} C_{i}^{\alpha /(1+\varepsilon)}\right)^{1+\varepsilon}\right]\right)^{(1+\varepsilon / 2) /(1+\varepsilon)}<\infty .
$$

The statement of the theorem with the first expression for $H$ will follow from Theorem 3.1 once we prove that condition (3.1) holds. To this end, define

$$
R^{*}=\sum_{i=1}^{N} C_{i} R_{i}+Q
$$

Then

$$
\begin{aligned}
\left|\mathrm{P}(R>t)-\mathrm{E}\left[\sum_{i=1}^{N} \mathbf{1}\left(C_{i} R_{i}>t\right)\right]\right| \leq & \left|\mathrm{P}(R>t)-\mathrm{P}\left(\max _{1 \leq i \leq N} C_{i} R_{i}>t\right)\right| \\
& +\left|\mathrm{P}\left(\max _{1 \leq i \leq N} C_{i} R_{i}>t\right)-\mathrm{E}\left[\sum_{i=1}^{N} \mathbf{1}\left(C_{i} R_{i}>t\right)\right]\right| .
\end{aligned}
$$


Since $R \stackrel{\mathrm{D}}{=} R^{*} \geq \max _{1 \leq i \leq N} C_{i} R_{i}$, the first absolute value disappears. For the second one, note that

$$
\begin{aligned}
& \mathrm{E}\left[\sum_{i=1}^{N} \mathbf{1}\left(C_{i} R_{i}>t\right)\right]-\mathrm{P}\left(\max _{1 \leq i \leq N} C_{i} R_{i}>t\right) \\
& \quad=\mathrm{E}\left[\sum_{i=1}^{N} \mathbf{1}\left(C_{i} R_{i}>t\right)\right]-\mathrm{E}\left[\mathbf{1}\left(\max _{1 \leq i \leq N} C_{i} R_{i}>t\right)\right] \\
& \quad \geq 0 .
\end{aligned}
$$

Now it follows that

$$
\begin{aligned}
\left|\mathrm{P}(R>t)-\mathrm{E}\left[\sum_{i=1}^{N} \mathbf{1}\left(C_{i} R_{i}>t\right)\right]\right| \leq & \mathrm{P}(R>t)-\mathrm{P}\left(\max _{1 \leq i \leq N} C_{i} R_{i}>t\right) \\
& +\mathrm{E}\left[\sum_{i=1}^{N} \mathbf{1}\left(C_{i} R_{i}>t\right)\right]-\mathrm{P}\left(\max _{1 \leq i \leq N} C_{i} R_{i}>t\right) .
\end{aligned}
$$

Note that the integral corresponding to (4.9) is finite by Lemma 4.6 if we show that the assumptions of Lemma 4.6 are satisfied when $\alpha>1$. Note that in this case we can choose $\varepsilon>0$ such that $\alpha /(1+\varepsilon) \geq 1$ and use the inequality

$$
\sum_{i=1}^{k} x_{i}^{\beta} \leq\left(\sum_{i=1}^{k} x_{i}\right)^{\beta}
$$

for $\beta \geq 1, x_{i} \geq 0$, and $k \leq \infty$ to obtain

$$
\mathrm{E}\left[\left(\sum_{i=1}^{N} C_{i}^{\alpha /(1+\varepsilon)}\right)^{1+\varepsilon}\right] \leq \mathrm{E}\left[\left(\sum_{i=1}^{N} C_{i}\right)^{\alpha}\right]<\infty
$$

Therefore, it only remains to show that

$$
\int_{0}^{\infty}\left(\mathrm{P}(R>t)-\mathrm{P}\left(\max _{1 \leq i \leq N} C_{i} R_{i}>t\right)\right) t^{\alpha-1} \mathrm{~d} t<\infty .
$$

To see this, note that $R \stackrel{\mathrm{D}}{=} R^{*}$ and $\mathbf{1}\left(R^{*}>t\right)-\mathbf{1}\left(\max _{1 \leq i \leq N} C_{i} R_{i}>t\right) \geq 0$, and, thus, by Fubini's theorem we have

$$
\int_{0}^{\infty}\left(\mathrm{P}(R>t)-\mathrm{P}\left(\max _{1 \leq i \leq N} C_{i} R_{i}>t\right)\right) t^{\alpha-1} \mathrm{~d} t=\frac{1}{\alpha} \mathrm{E}\left[\left(R^{*}\right)^{\alpha}-\left(\max _{1 \leq i \leq N} C_{i} R_{i}\right)^{\alpha}\right] .
$$

If $0<\alpha \leq 1$, we apply (4.10) to obtain

$$
\mathrm{E}\left[\left(R^{*}\right)^{\alpha}-\left(\max _{1 \leq i \leq N} C_{i} R_{i}\right)^{\alpha}\right] \leq \mathrm{E}\left[Q^{\alpha}+\sum_{i=1}^{N}\left(C_{i} R_{i}\right)^{\alpha}-\left(\max _{1 \leq i \leq N} C_{i} R_{i}\right)^{\alpha}\right]
$$

which is finite by Lemma 4.6 and the assumption that $\mathrm{E}\left[Q^{\alpha}\right]<\infty$. 
If $\alpha>1$, we have $\left(\sum_{i=1}^{k} x_{i}\right)^{\alpha} \geq \sum_{i=1}^{k} x_{i}^{\alpha}, x_{i} \geq 0, k \leq \infty$, implying that we can split the expectation as

$$
\begin{aligned}
\mathrm{E}\left[\left(R^{*}\right)^{\alpha}-\left(\max _{1 \leq i \leq N} C_{i} R_{i}\right)^{\alpha}\right]= & \mathrm{E}\left[\left(R^{*}\right)^{\alpha}-\sum_{i=1}^{N}\left(C_{i} R_{i}\right)^{\alpha}\right] \\
& +\mathrm{E}\left[\sum_{i=1}^{N}\left(C_{i} R_{i}\right)^{\alpha}-\left(\max _{1 \leq i \leq N} C_{i} R_{i}\right)^{\alpha}\right],
\end{aligned}
$$

which can be done since both expressions inside the expectations on the right-hand side are nonnegative. The first expectation is finite by Lemma 4.7 and the second expectation is again finite by Lemma 4.6.

Finally, applying Theorem 3.1 gives

$$
\mathrm{P}(R>t) \sim H t^{-\alpha},
$$

where $H=\left(\mathrm{E}\left[\sum_{j=1}^{N} C_{j}^{\alpha} \log C_{j}\right]\right)^{-1} \int_{0}^{\infty} v^{\alpha-1}\left(\mathrm{P}(R>v)-\mathrm{E}\left[\sum_{j=1}^{N} \mathbf{1}\left(C_{j} R>v\right)\right]\right) \mathrm{d} v$.

To obtain the second expression for $H$, note that

$$
\begin{aligned}
\int_{0}^{\infty} & v^{\alpha-1}\left(\mathrm{P}(R>v)-\mathrm{E}\left[\sum_{j=1}^{N} \mathbf{1}\left(C_{j} R>v\right)\right]\right) \mathrm{d} v \\
& =\int_{0}^{\infty} v^{\alpha-1} \mathrm{E}\left[\mathbf{1}\left(\sum_{i=1}^{N} C_{i} R_{i}+Q>v\right)-\sum_{i=1}^{N} \mathbf{1}\left(C_{i} R_{i}>v\right)\right] \mathrm{d} v \\
& =\mathrm{E}\left[\int_{0}^{\infty} v^{\alpha-1}\left(\mathbf{1}\left(\sum_{i=1}^{N} C_{i} R_{i}+Q>v\right)-\sum_{i=1}^{N} \mathbf{1}\left(C_{i} R_{i}>v\right)\right) \mathrm{d} v\right] \\
& =\mathrm{E}\left[\int_{0}^{\sum_{i=1}^{N} C_{i} R_{i}+Q} v^{\alpha-1} \mathrm{~d} v-\sum_{i=1}^{N} \int_{0}^{C_{i} R_{i}} v^{\alpha-1} \mathrm{~d} v\right] \\
& =\frac{1}{\alpha} \mathrm{E}\left[\left(\sum_{i=1}^{N} C_{i} R_{i}+Q\right)^{\alpha}-\sum_{i=1}^{N}\left(C_{i} R_{i}\right)^{\alpha}\right]
\end{aligned}
$$

where (4.12) is justified by Fubini's theorem and the integrability of

$$
\begin{aligned}
v^{\alpha-1} \mid & \mathbf{1}\left(\sum_{i=1}^{N} C_{i} R_{i}+Q>v\right)-\sum_{i=1}^{N} \mathbf{1}\left(C_{i} R_{i}>v\right) \mid \\
\leq & v^{\alpha-1}\left(\mathbf{1}\left(\sum_{i=1}^{N} C_{i} R_{i}+Q>v\right)-\mathbf{1}\left(\max _{1 \leq i \leq N} C_{i} R_{i}>v\right)\right) \\
& +v^{\alpha-1}\left(\sum_{i=1}^{N} \mathbf{1}\left(C_{i} R_{i}>v\right)-\mathbf{1}\left(\max _{1 \leq i \leq N} C_{i} R_{i}>v\right)\right),
\end{aligned}
$$

which is a consequence of (4.11) and Lemma 4.6; (4.13) follows from the observation that

$$
v^{\alpha-1} \mathbf{1}\left(\sum_{i=1}^{N} C_{i} R_{i}+Q>v\right) \text { and } v^{\alpha-1} \sum_{i=1}^{N} \mathbf{1}\left(C_{i} R_{i}>v\right)
$$

are each a.s. absolutely integrable with respect to $v$ as well.

This completes the proof. 
As indicated earlier, when $\alpha \geq 1$ is an integer, we can obtain the following explicit expression for $H$.

Corollary 4.1. For integer $\alpha \geq 1$, and under the same assumptions of Theorem 4.1, the constant $H$ can be explicitly computed as a function of $\mathrm{E}\left[R^{k}\right], \mathrm{E}\left[C^{k}\right]$, and $\mathrm{E}\left[Q^{k}\right], 0 \leq k \leq \alpha-1$. In particular, for $\alpha=1$,

$$
H=\frac{\mathrm{E}[Q]}{\mathrm{E}\left[\sum_{i=1}^{N} C_{i} \log C_{i}\right]}
$$

and, for $\alpha=2$,

$$
\begin{aligned}
H & =\frac{\mathrm{E}\left[Q^{2}\right]+2 \mathrm{E}[R] \mathrm{E}\left[Q \sum_{i=1}^{N} C_{i}\right]+2(\mathrm{E}[R])^{2} \mathrm{E}\left[\sum_{i=1}^{N} \sum_{j=i+1}^{N} C_{i} C_{j}\right]}{2 \mathrm{E}\left[\sum_{i=1}^{N} C_{i}^{2} \log C_{i}\right]}, \\
\mathrm{E}[R] & =\frac{\mathrm{E}[Q]}{1-\mathrm{E}\left[\sum_{i=1}^{N} C_{i}\right]} .
\end{aligned}
$$

Proof. The proof follows directly from multinomial expansions of the second expression for $H$ in Theorem 4.1 .

\subsection{The homogeneous recursion}

In this subsection we briefly describe how the methodology developed in the previous sections can be applied to study the critical, $\mathrm{E}\left[\sum_{i=1}^{N} C_{i}\right]=1$, homogeneous linear recursion

$$
R \stackrel{\mathrm{D}}{=} \sum_{i=1}^{N} C_{i} R_{i}
$$

where $\left(N, C_{1}, C_{2}, \ldots\right)$ is a nonnegative random vector with $N \in \mathbb{N} \cup\{\infty\}$ and $\left\{R_{i}\right\}_{i \in \mathbb{N}}$ is a sequence of i.i.d. random variables independent of $\left(N, C_{1}, C_{2}, \ldots\right)$ with the same distribution as $R$. This equation has been studied extensively in the literature under various different assumptions; for recent results, see [2], [17], [23], and the references therein.

Based on the model from Section 4 we can construct a solution to (4.14) as follows. Consider the process $\left\{W_{n}\right\}_{n \geq 0}$ defined by (4.2) with $Q_{i} \equiv 1$. Then, the $\left\{W_{n}\right\}$ satisfy in distribution the homogeneous recursion in (4.5) and, given that $\mathrm{E}\left[\sum_{i=1}^{N} C_{i}\right]=1$, we have $\mathrm{E}\left[W_{n}\right]=1$. Hence, $\left\{W_{n}\right\}_{n \geq 0}$ is a nonnegative martingale and, by the martingale convergence theorem, $W_{n} \rightarrow R$ a.s. with $\mathrm{E}[R] \leq 1$. Next, provided that

$$
\mathrm{E}\left[\sum_{i=1}^{N} C_{i} \log C_{i}\right]<0 \text { and } \mathrm{E}\left[\left(\sum_{i=1}^{N} C_{i}\right) \log ^{+}\left(\sum_{i=1}^{N} C_{i}\right)\right]<\infty,
$$

it can be shown that $\mathrm{E}[R]=1$; see Theorem 1.1(d) of [2] (see also Theorem 2 of [23]). Here $\log ^{+} x=\max (\log x, 0)$. Furthermore, as argued in Equation (1.9) of [2], it can easily be shown that this $R$ is a solution to (4.14). Note that the same construction of the solution $R$ on a branching tree was given in [2] and [23]. Since the solutions to (4.14) are scale invariant, this construction also shows that, for any $m>0$, there is a solution $R$ with mean $m$; or, equivalently, it is enough to study the solutions with mean 1 . Moreover, under additional assumptions, it can be shown that this constructed $R$ is the only solution with mean 1, see, e.g. [17], [22], and [23]. However, it is not the objective of this section to study the uniqueness of this solution, rather we focus on studying the tail behavior of any such possible solution (since our Theorem 3.1 does 
not require the uniqueness of $R$ ). As a side note, we point out that (4.14) can have solutions if $\mathrm{E}\left[\sum_{i=1}^{N} C_{i}^{\beta}\right]=1$ for some $0<\beta<1$, as studied in [17] and [22].

A version of the following theorem, with a possibly less explicit constant, was previously proved in Theorem 2.2 of [23] and Proposition 7 of [17]; they also studied the lattice case. Regarding the lattice case, as pointed out earlier in the remark after Theorem 3.1, all the results in this paper can be developed for this case as well by using the corresponding renewal theorem.

Theorem 4.2. Suppose that there exists $j \geq 1$ with $\mathrm{P}\left(N \geq j, C_{j}>0\right)>0$ such that the measure $\mathrm{P}\left(\log C_{j} \in \mathrm{d} u, C_{j}>0, N \geq j\right)$ is nonarithmetic. Suppose further that, for some $\alpha>1$, $\mathrm{E}\left[\left(\sum_{i=1}^{N} C_{i}\right)^{\alpha}\right]<\infty, \quad \mathrm{E}\left[\sum_{i=1}^{N} C_{i}^{\alpha} \log ^{+} C_{i}\right]<\infty$, and $\mathrm{E}\left[\sum_{i=1}^{N} C_{i}\right]=\mathrm{E}\left[\sum_{i=1}^{N} C_{i}^{\alpha}\right]=1$. Then, (4.14) has a solution $R$ with $0<\mathrm{E}[R]<\infty$ such that

$$
\mathrm{P}(R>t) \sim H t^{-\alpha} \text { as } t \rightarrow \infty
$$

where $0 \leq H<\infty$ is given by

$$
\begin{aligned}
H & =\frac{1}{\mathrm{E}\left[\sum_{i=1}^{N} C_{i}^{\alpha} \log C_{i}\right]} \int_{0}^{\infty} v^{\alpha-1}\left(\mathrm{P}(R>v)-\mathrm{E}\left[\sum_{i=1}^{N} \mathbf{1}\left(C_{i} R>v\right)\right]\right) \mathrm{d} v \\
& =\frac{\mathrm{E}\left[\left(\sum_{i=1}^{N} C_{i} R_{i}\right)^{\alpha}-\sum_{i=1}^{N}\left(C_{i} R_{i}\right)^{\alpha}\right]}{\alpha \mathrm{E}\left[\sum_{i=1}^{N} C_{i}^{\alpha} \log C_{i}\right]} .
\end{aligned}
$$

Furthermore, if $\mathrm{P}(\tilde{N} \geq 2)>0, \tilde{N}=\sum_{i=1}^{N} \mathbf{1}\left(C_{i}>0\right)$, then $H>0$.

Proof. By the assumptions, the function $\varphi(\theta):=\mathrm{E}\left[\sum_{i=1}^{N} C_{j}^{\theta}\right]$ is convex, finite, and continuous on $[1, \alpha]$, since $\varphi(1)=\varphi(\alpha)=1$. Furthermore, by standard arguments, it can be shown that both $\varphi^{\prime}(\theta)$ and $\varphi^{\prime \prime}(\theta)$ exist on the open interval $(1, \alpha)$ and, in particular,

$$
\varphi^{\prime \prime}(\theta)=\mathrm{E}\left[\sum_{i=1}^{N} C_{i}^{\theta}\left(\log C_{i}\right)^{2}\right] .
$$

Clearly, $\varphi^{\prime \prime}(\theta)>0$ provided that $\mathrm{P}\left(C_{i} \in\{0,1\}, 1 \leq i \leq N\right)<1$. To see that this is indeed the case, note that $\mathrm{E}\left[\sum_{i=1}^{N} C_{i}\right]=1$ implies that $\mathrm{P}\left(C_{i} \equiv 0,1 \leq i \leq N\right)<1$, which, combined with the nonarithmetic assumption, yields $\mathrm{P}\left(C_{i} \in\{0,1\}, 1 \leq i \leq N\right)<1$. Hence, there exist $1<\theta_{1}<\theta_{2}<\alpha$ such that $\varphi^{\prime}\left(\theta_{1}\right)<0$ and $\varphi^{\prime}\left(\theta_{2}\right)>0$, implying by the monotonicity of $\varphi^{\prime}(\cdot)$ and monotone convergence that

$$
\begin{gathered}
0<\varphi^{\prime}(\alpha-)=\mathrm{E}\left[\sum_{i=1}^{N} C_{i}^{\alpha} \log C_{i}\right] \leq \mathrm{E}\left[\sum_{i=1}^{N} C_{i}^{\alpha} \log ^{+} C_{i}\right]<\infty \\
\text { and } \varphi^{\prime}(1+)=\mathrm{E}\left[\sum_{i=1}^{N} C_{i} \log C_{i}\right]<0 .
\end{gathered}
$$

From the last expression and the observation $\mathrm{E}\left[\left(\sum_{i=1}^{N} C_{i}\right) \log ^{+}\left(\sum_{i=1}^{N} C_{i}\right)\right]<\infty$ (implied by $\left.\mathrm{E}\left[\left(\sum_{i=1}^{N} C_{i}\right)^{\alpha}\right]<\infty\right)$ it follows, as argued at the beginning of this section, that recursion (4.14) has a solution with finite positive mean; see Theorem 1.1(d) and Equation (1.9) of [2] (see also Theorem 2 of [23]).

Next, in order to apply Theorem 3.1, we use (4.15) and $\mathrm{E}\left[R^{\beta}\right]<\infty$ for any $0<\beta<\alpha$; the latter follows from Theorem 3.1 of [2] and the strict convexity of $\varphi(\cdot)$ argued above (see also 
Proposition 4 of [17] and Theorem 2.1 of [23]). The rest of the proof, except for the $H>0$ part, proceeds exactly as that of Theorem 4.1 by setting $Q \equiv 0$.

Regarding the $H>0$ statement, the assumption that $\mathrm{P}(\tilde{N} \geq 2)>0$ implies that there exist $1 \leq n \leq \infty$ and $1 \leq i_{1}<i_{2}<n+1$ such that $\mathrm{P}\left(N=n, C_{i_{1}}>0, C_{i_{2}}>0\right)>0$, which further yields, for some $\delta>0$,

$$
\mathrm{P}\left(N \geq i_{2}, C_{i_{1}}>\delta, C_{i_{2}}>\delta\right)>0 .
$$

Next, by using the inequality $\left(x_{1}+x_{2}\right)^{\alpha} \geq x_{1}^{\alpha}+x_{2}^{\alpha}$ for $x_{1}, x_{2} \geq 0$ and $\alpha>1$, the second expressions for $H$ in the theorem can be bounded from below by

$$
H \geq \frac{\mathrm{E}\left[\mathbf{1}\left(N \geq i_{2}\right)\left(\left(C_{i_{1}} R_{i_{1}}+C_{i_{2}} R_{i_{2}}\right)^{\alpha}-\left(C_{i_{1}} R_{i_{1}}\right)^{\alpha}-\left(C_{i_{2}} R_{i_{2}}\right)^{\alpha}\right)\right]}{\alpha \mathrm{E}\left[\sum_{i=1}^{N} C_{i}^{\alpha} \log C_{i}\right]} .
$$

To further bound the numerator in (4.17), we define the function

$$
f(x)=(1+x)^{\alpha}-1-x^{\alpha}-c x^{\alpha-\varepsilon},
$$

where $0<\varepsilon<\alpha-1,0<c<2^{\gamma}-1$, and $\gamma=\alpha-1-\varepsilon$. It can be shown that $f(x) \geq 0$ for $x \in[0,1]$, since $f(0)=0$ and $f^{\prime}(x) \geq \alpha x^{\gamma}\left((1+1 / x)^{\gamma}-1-c\right) \geq 0$ on $[0,1]$. Hence, by using the inequality $f(x) \geq 0$, we derive, for $x_{1} \geq 0, x_{2} \geq 0, \max \left\{x_{1}, x_{2}\right\}>0$, and $x=\min \left\{x_{1}, x_{2}\right\} / \max \left\{x_{1}, x_{2}\right\}$,

$$
\begin{aligned}
\left(x_{1}+x_{2}\right)^{\alpha}-x_{1}^{\alpha}-x_{2}^{\alpha} & =\left(\max \left\{x_{1}, x_{2}\right\}\right)^{\alpha}\left((1+x)^{\alpha}-1-x^{\alpha}\right) \\
& \geq c\left(\max \left\{x_{1}, x_{2}\right\}\right)^{\alpha} x^{\alpha-\varepsilon} \\
& \geq c\left(\min \left\{x_{1}, x_{2}\right\}\right)^{\alpha}
\end{aligned}
$$

the inequality clearly holds even if $\max \left\{x_{1}, x_{2}\right\}=0$ since both of its sides are zero. Thus, by applying this last inequality to (4.17) and using (4.16), we obtain

$$
\begin{aligned}
H & \geq \frac{c \mathrm{E}\left[\mathbf{1}\left(N \geq i_{2}\right)\left(\min \left\{C_{i_{1}} R_{i_{1}}, C_{i_{2}} R_{i_{2}}\right\}\right)^{\alpha}\right]}{\alpha \mathrm{E}\left[\sum_{i=1}^{N} C_{i}^{\alpha} \log C_{i}\right]} \\
& \geq \frac{c \delta^{\alpha} \mathrm{P}\left(N \geq i_{2}, C_{i_{1}}>\delta, C_{i_{2}}>\delta\right) \mathrm{E}\left[\left(\min \left\{R_{i_{1}}, R_{i_{2}}\right\}\right)^{\alpha}\right]}{\alpha \mathrm{E}\left[\sum_{i=1}^{N} C_{i}^{\alpha} \log C_{i}\right]} \\
& >0 .
\end{aligned}
$$

This completes the proof.

Remarks. (i) Note that the assumptions of Theorem 4.2 differ slightly from those of Theorem 4.1 in the condition

$$
0<\mathrm{E}\left[\sum_{i=1}^{N} C_{i}^{\alpha} \log C_{i}\right]<\infty
$$

which is implied by $\mathrm{E}\left[\sum_{i=1}^{N} C_{i}^{\alpha} \log ^{+} C_{i}\right]<\infty$, the strict convexity of $\varphi(\theta)=\mathrm{E}\left[\sum_{i=1}^{N} C_{i}^{\theta}\right]$ and the hypothesis that $\varphi(1)=\varphi(\alpha)=1$, as argued in the preceding proof.

(ii) The assumption that $\mathrm{P}(\tilde{N} \geq 2)>0$ is the minimal one to ensure the existence of a nontrivial solution; see conditions (H0) of [22] and (C4) of [2]. Otherwise, when $\mathrm{P}(\tilde{N} \leq 1)=1, W_{n}$ is a simple multiplicative random walk with no branching; clearly, in this case our expression for 
$H$ reduces to 0 . Also, if $\mathrm{P}\left(\sum_{i=1}^{N} C_{i}=1\right)=1, R$ can only be a constant; see the remark above Theorem 2.1 of [23]. However, this last case is excluded from the theorem since $\mathrm{P}\left(\sum_{i=1}^{N} C_{i}=1\right)=1$ implies that $C_{i} \leq 1$ a.s., which, in conjunction with $\varphi(\alpha)=1, \alpha>1$, yields $\mathrm{P}\left(C_{i} \in\{0,1\}, 1 \leq i \leq N\right)=1$, but this cannot happen due to the nonarithmetic assumption.

(iii) Note also that condition (C3) of [2] (equivalent to $\mathrm{P}\left(C_{i} \in\{0,1\}, 1 \leq i \leq N\right)<1$ in our notation) is implied by the nonarithmetic assumption of our theorem. Interestingly enough, if this last condition fails, Lemma 1.1 of [22] shows that (4.14) has no nontrivial solutions.

(iv) As stated earlier, a version of Theorem 4.2 was proved in Theorem 2.2 of [23] by transforming recursion (4.14) into a first-order difference (autoregressive/perpetuity) equation on a different probability space; see Lemma 4.1 of [23]. However, it appears that the method from [23] does not extend to the nonhomogeneous and nonlinear problems that we cover here, since the proof of Lemma 4.1 of [23] critically depends on having both $\mathrm{E}[R]=1$ and $\mathrm{E}\left[\sum_{i=1}^{N} C_{i}\right]=1$.

Similarly as in Corollary 4.1, the constant $H$ can be computed explicitly for an integer $\alpha \geq 2$.

Corollary 4.2. For an integer $\alpha \geq 2$, and under the same assumptions of Theorem 4.2, the constant $H$ can be explicitly computed as a function of $\mathrm{E}\left[R^{k}\right]$ and $\mathrm{E}\left[C^{k}\right], 1 \leq k \leq \alpha-1$. In particular, for $\alpha=2$,

$$
H=\frac{\mathrm{E}\left[\sum_{i=1}^{N} \sum_{j=i+1}^{N} C_{i} C_{j}\right]}{\mathrm{E}\left[\sum_{i=1}^{N} C_{i}^{2} \log C_{i}\right]} .
$$

Proof. The proof follows directly from multinomial expansions of the second expression for $H$ in Theorem 4.2 .

We also want to point out that, for a general noninteger $\alpha>1$, we can use Lemma 4.1 to obtain the following bound for $H$ :

$$
H \leq \frac{\left(\mathrm{E}\left[R^{p-1}\right]\right)^{\alpha /(p-1)} \mathrm{E}\left[\left(\sum_{i=1}^{N} C_{i}\right)^{\alpha}\right]}{\alpha \mathrm{E}\left[\sum_{i=1}^{N} C_{i}^{\alpha} \log C_{i}\right]} .
$$

Here $p=\lceil\alpha\rceil$.

\section{The maximum recursion: $R=\left(\bigvee_{i=1}^{N} C_{i} R_{i}\right) \vee Q$}

In order to show the general applicability of the implicit renewal theorem, we study in this section the nonlinear recursion

$$
R \stackrel{\mathrm{D}}{=}\left(\bigvee_{i=1}^{N} C_{i} R_{i}\right) \vee Q
$$

where $\left(Q, N, C_{1}, C_{2}, \ldots\right)$ is a nonnegative random vector with $N \in \mathbb{N} \cup\{\infty\}, \mathrm{P}(Q>0)>0$, and $\left\{R_{i}\right\}_{i \in \mathbb{N}}$ is a sequence of i.i.d. random variables independent of $\left(Q, N, C_{1}, C_{2}, \ldots\right)$ with the same distribution as $R$. Note that in the case of page-ranking applications, where the $\left\{R_{i}\right\}$ represent the ranks of the neighboring pages, the potential ranking algorithm defined by the preceding recursion determines the rank of a page as a weighted version of the most highly ranked neighboring page. In other words, the highest ranked reference has the dominant impact. Similarly to the homogeneous linear case, this recursion was previously studied in [5] under 
the assumptions that $Q \equiv 0, N=\infty$, and the $\left\{C_{i}\right\}$ are real-valued deterministic constants. The more closely related case of $Q \equiv 0$ and $\left\{C_{i}\right\} \geq 0$ being random was studied earlier in [18]. Furthermore, these max-type stochastic recursions appear in a wide variety of applications, ranging from the average case analysis of algorithms to statistical physics; see [1] for a recent survey.

Using standard arguments, we start by constructing a solution to (5.1) on a tree and then we show that this solution is finite a.s. and unique under iterations and some moment conditions, similarly to what was done for the nonhomogeneous linear recursion in Section 4. Our main result of this section is stated in Theorem 5.1.

Using the same notation as in Section 4, define the process

$$
V_{n}=\bigvee_{i \in A_{n}} Q_{i} \Pi_{i}, \quad n \geq 0,
$$

on the weighted branching tree $\mathcal{T}_{Q, C}$, as constructed in Section 2. Recall that the convention is that $\left(Q, N, C_{1}, C_{2}, \ldots\right)=\left(Q_{\varnothing}, N_{\varnothing}, C_{(\varnothing, 1)}, C_{(\varnothing, 2)}, \ldots\right)$ denotes the random vector corresponding to the root node.

With a possible abuse of notation relative to Section 4 , define the process $\left\{R^{(n)}\right\}_{n \geq 0}$ according to

$$
R^{(n)}=\bigvee_{k=0}^{n} V_{k}, \quad n \geq 0 .
$$

Just as with the linear recursion from Section 4 , it is not hard to see that $R^{(n)}$ satisfies the recursion

$$
R^{(n)}=\left(\bigvee_{j=1}^{N_{\varnothing}} C_{(\varnothing, j)} R_{j}^{(n-1)}\right) \vee Q_{\varnothing}=\left(\bigvee_{j=1}^{N} C_{j} R_{j}^{(n-1)}\right) \vee Q,
$$

where $\left\{R_{j}^{(n-1)}\right\}$ are independent copies of $R^{(n-1)}$ corresponding to the tree starting with individual $j$ in the first generation and ending on the $n$th generation. We can also verify that

$$
V_{n}=\bigvee_{k=1}^{N_{\varnothing}} C_{(\varnothing, k)} \bigvee_{\left(k, \ldots, i_{n}\right) \in A_{n}} Q_{\left(k, \ldots, i_{n}\right)} \prod_{j=2}^{n} C_{\left(k, \ldots, i_{j}\right)} \stackrel{\mathrm{D}}{=} \bigvee_{k=1}^{N} C_{k} V_{(n-1), k},
$$

where $\left\{V_{(n-1), k}\right\}$ is a sequence of i.i.d. random variables independent of $\left(N, C_{1}, C_{2}, \ldots\right)$ and having the same distribution as $V_{n-1}$.

We now define the random variable $R$ according to

$$
R:=\lim _{n \rightarrow \infty} R^{(n)}=\bigvee_{k=0}^{\infty} V_{k}
$$

Note that $R^{(n)}$ is monotone increasing sample pathwise, so $R$ is well defined. Also, by the monotonicity of $R^{(n)},(5.3)$, and monotone convergence, we find that $R$ solves

$$
R=\left(\bigvee_{j=1}^{N_{\varnothing}} C_{(\varnothing, j)} R_{j}^{(\infty)}\right) \vee Q_{\varnothing}=\left(\bigvee_{j=1}^{N} C_{j} R_{j}^{(\infty)}\right) \vee Q
$$

where $\left\{R_{j}^{(\infty)}\right\}_{j \in \mathbb{N}}$ are i.i.d. copies of $R$, independent of $\left(Q, N, C_{1}, C_{2}, \ldots\right)$. Clearly, this implies that $R$, as defined by (5.4), is a solution in distribution to (5.1). However, this solution 
might be $\infty$. Now, we establish the finiteness of the moments of $R$ and, in particular, the fact that $R<\infty$ a.s. in the following lemma; its proof uses standard contraction arguments, but is included for completeness.

Lemma 5.1. Assume that $\rho_{\beta}=\mathrm{E}\left[\sum_{i=1}^{N} C_{i}^{\beta}\right]<1$ and $\mathrm{E}\left[Q^{\beta}\right]<\infty$ for some $\beta>0$. Then, $\mathrm{E}\left[R^{\gamma}\right]<\infty$ for all $0<\gamma \leq \beta$, and, in particular, $R<\infty$ a.s. Moreover, if $\beta \geq 1, R^{(n)} \stackrel{L_{\beta}}{\rightarrow} R$, where $L_{\beta}$ stands for convergence in the $\left(\mathrm{E}|\cdot|^{\beta}\right)^{1 / \beta}$ norm.

Proof. By following the same steps leading to (3.6), we obtain, for any $k \geq 0$,

$$
\mathrm{E}\left[V_{k}^{\beta}\right]=\mathrm{E}\left[\bigvee_{i \in A_{k}} Q_{i}^{\beta} \Pi_{i}^{\beta}\right] \leq \mathrm{E}\left[\sum_{i \in A_{k}} Q_{i}^{\beta} \Pi_{i}^{\beta}\right]=\mathrm{E}\left[Q^{\beta}\right] \rho_{\beta}^{k}
$$

Hence,

$$
\mathrm{E}\left[R^{\beta}\right]=\mathrm{E}\left[\bigvee_{k=0}^{\infty} V_{k}^{\beta}\right] \leq \mathrm{E}\left[\sum_{k=0}^{\infty} V_{k}^{\beta}\right] \leq \frac{\mathrm{E}\left[Q^{\beta}\right]}{1-\rho_{\beta}}<\infty,
$$

implying that $\mathrm{E}\left[R^{\gamma}\right]<\infty$ for all $0<\gamma \leq \beta$.

That $R^{(n)} \stackrel{L_{\beta}}{\longrightarrow} R$ whenever $\beta \geq 1$ follows from noting that

$$
\mathrm{E}\left[\left|R^{(n)}-R\right|^{\beta}\right] \leq \mathrm{E}\left[\left(\bigvee_{k=n+1}^{\infty} V_{k}\right)^{\beta}\right] \leq \mathrm{E}\left[\sum_{k=n+1}^{\infty} V_{k}^{\beta}\right]
$$

and applying the preceding geometric bound for $\mathrm{E}\left[V_{k}^{\beta}\right]$.

Just as with the linear recursion from Section 4 , we can define the process $\left\{R_{n}^{*}\right\}$ as

$$
R_{n}^{*}:=R^{(n-1)} \vee V_{n}\left(R_{0}^{*}\right), \quad n \geq 1,
$$

where

$$
V_{n}\left(R_{0}^{*}\right)=\bigvee_{i \in A_{n}} R_{0, i}^{*} \Pi_{i}
$$

and $\left\{R_{0, i}^{*}\right\}_{i \in U}$ are i.i.d. copies of an initial value $R_{0}^{*}$, independent of the entire weighted tree $\mathcal{T}_{Q, C}$. It follows from (5.3) and (5.6) that

$$
R_{n+1}^{*}=\bigvee_{j=1}^{N} C_{j}\left(R_{j}^{(n-1)} \vee \sum_{i \in A_{n, j}} R_{0, i}^{*} \prod_{k=2}^{n} C_{\left(j, \ldots, i_{k}\right)}\right)+Q=\bigvee_{j=1}^{N} C_{j} R_{n, j}^{*} \vee Q,
$$

where $\left\{R_{j}^{(n-1)}\right\}$ are independent copies of $R^{(n-1)}$ corresponding to the tree starting with individual $j$ in the first generation and ending on the $n$th generation, and $A_{n, j}$ is the set of all nodes in the $(n+1)$ th generation that are descendants of individual $j$ in the first generation. Moreover, $\left\{R_{n, j}^{*}\right\}$ are i.i.d. copies of $R_{n}^{*}$, and, thus, $R_{n}^{*}$ is equal in distribution to the process obtained by iterating (5.1) with an initial condition $R_{0}^{*}$. This process can be shown to converge in distribution to $R$ for any initial condition $R_{0}^{*}$ satisfying the following moment condition.

Lemma 5.2. For any $R_{0}^{*} \geq 0$, if $\mathrm{E}\left[Q^{\beta}\right], \mathrm{E}\left[\left(R_{0}^{*}\right)^{\beta}\right]<\infty$, and $\rho_{\beta}<1$ for some $\beta>0$, then

$$
R_{n}^{*} \Rightarrow R
$$

with $\mathrm{E}\left[R^{\beta}\right]<\infty$. Furthermore, under these assumptions, the distribution of $R$ is the unique solution with finite $\beta$-moment to recursion (5.1). 
Proof. The result will follow from Slutsky's theorem (see Theorem 25.4 of [10, p. 332]) once we show that $V_{n}\left(R_{0}^{*}\right) \Rightarrow 0$. To this end, recall that $V_{n}\left(R_{0}^{*}\right)$ is the same as $V_{n}$ if we substitute the $Q_{i}$ by the $R_{0, i}^{*}$. Then, for every $\varepsilon>0$, we have

$$
\mathrm{P}\left(V_{n}\left(R_{0}^{*}\right)>\varepsilon\right) \leq \varepsilon^{-\beta} \mathrm{E}\left[V_{n}\left(R_{0}^{*}\right)^{\beta}\right] \leq \varepsilon^{-\beta} \rho_{\beta}^{n} \mathrm{E}\left[\left(R_{0}^{*}\right)^{\beta}\right] \quad(\text { by }(5.5)) .
$$

Since, by assumption, the right-hand side converges to 0 as $n \rightarrow \infty$, then $R_{n}^{*} \Rightarrow R$. Furthermore, $\mathrm{E}\left[R^{\beta}\right]<\infty$ by Lemma 5.1. Clearly, under the assumptions, the distribution of $R$ represents the unique solution to (5.1), since any other possible solution with finite $\beta$-moment would have to converge to the same limit.

Now we are ready to state the main result of this section.

Theorem 5.1. Let $\left(Q, N, C_{1}, C_{2}, \ldots\right)$ be a nonnegative random vector, with $N \in \mathbb{N} \cup\{\infty\}$ and $\mathrm{P}(Q>0)>0$, and let $R$ be the solution to (5.1) given by (5.4). Suppose that there exists $j \geq 1$ with $\mathrm{P}\left(N \geq j, C_{j}>0\right)>0$ such that the measure $\mathrm{P}\left(\log C_{j} \in \mathrm{d} u, C_{j}>0, N \geq j\right)$ is nonarithmetic, and that, for some $\alpha>0, \mathrm{E}\left[Q^{\alpha}\right]<\infty, 0<\mathrm{E}\left[\sum_{i=1}^{N} C_{i}^{\alpha} \log C_{i}\right]<\infty$, and $\mathrm{E}\left[\sum_{i=1}^{N} C_{i}^{\alpha}\right]=1$. In addition, assume that

(a) $\mathrm{E}\left[\left(\sum_{i=1}^{N} C_{i}\right)^{\alpha}\right]<\infty$ if $\alpha>1$, or

(b) $\mathrm{E}\left[\left(\sum_{i=1}^{N} C_{i}^{\alpha /(1+\varepsilon)}\right)^{1+\varepsilon}\right]<\infty$ for some $0<\varepsilon<1$ if $0<\alpha \leq 1$.

Then

$$
\mathrm{P}(R>t) \sim H t^{-\alpha} \quad \text { as } t \rightarrow \infty,
$$

where $0 \leq H<\infty$ is given by

$$
\begin{aligned}
H & =\frac{1}{\mathrm{E}\left[\sum_{i=1}^{N} C_{i}^{\alpha} \log C_{i}\right]} \int_{0}^{\infty} v^{\alpha-1}\left(\mathrm{P}(R>v)-\mathrm{E}\left[\sum_{i=1}^{N} \mathbf{1}\left(C_{i} R>v\right)\right]\right) \mathrm{d} v \\
& =\frac{\mathrm{E}\left[\left(\bigvee_{i=1}^{N} C_{i} R_{i}\right)^{\alpha} \vee Q^{\alpha}-\sum_{i=1}^{N}\left(C_{i} R_{i}\right)^{\alpha}\right]}{\alpha \mathrm{E}\left[\sum_{i=1}^{N} C_{i}^{\alpha} \log C_{i}\right]} .
\end{aligned}
$$

Proof. By Lemma 5.1 we know that $\mathrm{E}\left[R^{\beta}\right]<\infty$ for any $0<\beta<\alpha$. By the same arguments used in the proof of Theorem 4.1 we obtain $\mathrm{E}\left[\sum_{i=1}^{N} C_{i}^{\gamma}\right]<\infty$ for some $0 \leq \gamma<\alpha$. The statement of the theorem with the first expression for $H$ will follow from Theorem 3.1 once we prove that condition (3.1) holds. Define

$$
R^{*}=\left(\bigvee_{i=1}^{N} C_{i} R_{i}\right) \vee Q .
$$

Then,

$$
\begin{aligned}
\left|\mathrm{P}(R>t)-\mathrm{E}\left[\sum_{i=1}^{N} \mathbf{1}\left(C_{i} R_{i}>t\right)\right]\right| \leq & \left|\mathrm{P}(R>t)-\mathrm{P}\left(\max _{1 \leq i \leq N} C_{i} R_{i}>t\right)\right| \\
& +\left|\mathrm{P}\left(\max _{1 \leq i \leq N} C_{i} R_{i}>t\right)-\mathrm{E}\left[\sum_{i=1}^{N} \mathbf{1}\left(C_{i} R_{i}>t\right)\right]\right| .
\end{aligned}
$$

Since $R \stackrel{\mathrm{D}}{=} R^{*} \geq \max _{1 \leq i \leq N} C_{i} R_{i}$, the first absolute value disappears. The integral corresponding to the second term is finite by Lemma 4.6, just as in the proof of Theorem 4.1. To see that 
the integral corresponding to the first term,

$$
\int_{0}^{\infty}\left(\mathrm{P}(R>t)-\mathrm{P}\left(\max _{1 \leq i \leq N} C_{i} R_{i}>t\right)\right) t^{\alpha-1} \mathrm{~d} t,
$$

is finite, we proceed as in the proof of Theorem 4.1. First we use Fubini's theorem to obtain

$$
\begin{aligned}
\int_{0}^{\infty}\left(\mathrm{P}(R>t)-\mathrm{P}\left(\max _{1 \leq i \leq N} C_{i} R_{i}>t\right)\right) t^{\alpha-1} \mathrm{~d} t & =\frac{1}{\alpha} \mathrm{E}\left[\left(R^{*}\right)^{\alpha}-\left(\max _{1 \leq i \leq N} C_{i} R_{i}\right)^{\alpha}\right] \\
& =\frac{1}{\alpha} \mathrm{E}\left[\left(\bigvee_{i=1}^{N} C_{i} R_{i}\right)^{\alpha} \vee Q^{\alpha}-\left(\bigvee_{i=1}^{N} C_{i} R_{i}\right)^{\alpha}\right] \\
& \leq \frac{\mathrm{E}\left[Q^{\alpha}\right]}{\alpha}
\end{aligned}
$$

Now, applying Theorem 3.1 gives $\mathrm{P}(R>t) \sim H t^{-\alpha}$, where $H=\left(\mathrm{E}\left[\sum_{j=1}^{N} C_{j}^{\alpha} \log C_{j}\right]\right)^{-1} \times$ $\int_{0}^{\infty} v^{\alpha-1}\left(\mathrm{P}(R>v)-\mathrm{E}\left[\sum_{j=1}^{N} \mathbf{1}\left(C_{j} R>v\right)\right]\right) \mathrm{d} v$.

The same steps used in the proof of Theorem 4.1 give the second expression for $H$.

\section{Other recursions and concluding remarks}

As an illustration of the generality of the methodology presented in this paper, we mention in this section other recursions that fall within its scope. One example that is closely related to the recursions from Sections 4 and 5 is

$$
R \stackrel{\mathrm{D}}{=}\left(\bigvee_{i=1}^{N} C_{i} R_{i}\right)+Q
$$

where $\left(Q, N, C_{1}, C_{2}, \ldots\right)$ is a nonnegative vector with $N \in \mathbb{N} \cup\{\infty\}, \mathrm{P}(Q>0)>0$, and $\left\{R_{i}\right\}_{i \in \mathbb{N}}$ is a sequence of i.i.d. random variables independent of $\left(Q, N, C_{1}, C_{2}, \ldots\right)$ with the same distribution as $R$. Recursion (6.1) was termed 'discounted tree sums' in [1]; for additional details on the existence and uniqueness of its solution, see Section 4.4 of [1].

Similarly as in [14], it appears that one could study other nonlinear recursions on trees using implicit renewal theory. For example, one could analyze the solution to the equation

$$
R \stackrel{\mathrm{D}}{=} \sum_{i=1}^{N}\left(C_{i} R_{i}+B_{i} \sqrt{R_{i}}\right)+Q
$$

where $\left(Q, N, C_{1}, C_{2}, \ldots\right)$ is a nonnegative vector with $N \in \mathbb{N} \cup\{\infty\}, \mathrm{P}(Q>0)>0$, and $\left\{R, R_{i}\right\}_{i \geq 1}$ is a sequence of i.i.d. random variables independent of $\left(Q, N, C_{1}, C_{2}, \ldots\right)$. Here, the primary difficulty would be in establishing the existence and uniqueness of the solution as well as the finiteness of the moments.

\section{Proofs}

\subsection{Implicit renewal theorem on trees}

We give in this section the proof of Lemma 3.1. 
Proof of Lemma 3.1. Observe that the measure $\mathrm{E}\left[\sum_{i=1}^{N} \mathbf{1}\left(\log C_{i} \in \mathrm{d} u, C_{i}>0\right)\right]$ is nonarithmetic (nonlattice) by our assumption since if we assume to the contrary that it is lattice on a lattice set $L$ then on the complement $L^{\mathrm{c}}$ of this set we have

$$
0=\mathrm{E}\left[\sum_{i=1}^{N} \mathbf{1}\left(\log C_{i} \in L^{\mathrm{c}}, C_{i}>0\right)\right] \geq \mathrm{P}\left(\log C_{j} \in L^{\mathrm{c}}, C_{j}>0, N \geq j\right)>0,
$$

which is a contradiction. Hence, $\eta$ is nonarithmetic as well, and it places no mass at $-\infty$ due to the exponential term $\mathrm{e}^{\alpha u}$. To see that it is a probability measure, note that

$$
\begin{aligned}
\int_{-\infty}^{\infty} \eta(\mathrm{d} u) & =\int_{-\infty}^{\infty} \mathrm{e}^{\alpha u} \mathrm{E}\left[\sum_{j=1}^{N} \mathbf{1}\left(\log C_{j} \in \mathrm{d} u\right)\right] \\
& =\mathrm{E}\left[\sum_{j=1}^{N} \int_{-\infty}^{\infty} \mathrm{e}^{\alpha u} \mathbf{1}\left(\log C_{j} \in \mathrm{d} u\right)\right] \quad \text { (by Fubini's theorem) } \\
& =\mathrm{E}\left[\sum_{j=1}^{N} C_{j}^{\alpha}\right] \\
& =1 .
\end{aligned}
$$

Similarly, its mean is given by

$$
\int_{-\infty}^{\infty} u \eta(\mathrm{d} u)=\mathrm{E}\left[\sum_{j=1}^{N} C_{j}^{\alpha} \log C_{j}\right]
$$

To show that $\mu_{n}=\eta^{* n}$, we proceed by induction. Let $\mathcal{F}_{n}$ denote the $\sigma$-algebra generated by $\left\{\left(N_{i}, C_{(i, 1)}, C_{(i, 2)}, \ldots\right): i \in A_{j}, 0 \leq j \leq n-1\right\}, \mathcal{F}_{0}=\sigma(\varnothing, \Omega)$, and, for each $i \in A_{n}$, set $V_{i}=\log \Pi_{i}$. Hence, using this notation, we derive

$$
\begin{aligned}
\mu_{n+1}((-\infty, t]) & =\int_{-\infty}^{t} \mathrm{e}^{\alpha u} \mathrm{E}\left[\sum_{i \in A_{n}} \sum_{j=1}^{N_{i}} \mathbf{1}\left(V_{i}+\log C_{(i, j)} \in \mathrm{d} u\right)\right] \\
& =\int_{-\infty}^{t} \mathrm{e}^{\alpha u} \mathrm{E}\left[\sum_{i \in A_{n}} \mathrm{E}\left[\sum_{j=1}^{N_{i}} \mathbf{1}\left(V_{i}+\log C_{(i, j)} \in \mathrm{d} u\right) \mid \mathcal{F}_{n}\right]\right] \\
& =\mathrm{E}\left[\sum_{i \in A_{n}} \mathrm{e}^{\alpha V_{i}} \int_{-\infty}^{t} \mathrm{e}^{\alpha\left(u-V_{i}\right)} \mathrm{E}\left[\sum_{j=1}^{N_{i}} \mathbf{1}\left(\log C_{(i, j)} \in \mathrm{d} u-V_{i}\right) \mid \mathcal{F}_{n}\right]\right] \\
& =\mathrm{E}\left[\sum_{i \in A_{n}} \mathrm{e}^{\alpha V_{i}} \eta\left(\left(-\infty, t-V_{i}\right]\right)\right] \\
& =\int_{-\infty}^{\infty} \eta((-\infty, t-v]) \mu_{n}(\mathrm{~d} v),
\end{aligned}
$$

where in the fourth equality we used the independence of $\left(N_{i}, C_{(i, 1)}, C_{(i, 2)}, \ldots\right)$ from $\mathcal{F}_{n}$. Therefore, $\mu_{n+1}(\mathrm{~d} t)=\left(\eta * \mu_{n}\right)(\mathrm{d} t)$ and the induction hypothesis gives the result. 


\subsection{Moments of $W_{n}$}

In this section we prove Lemmas 4.1, 4.2, and 4.3. We also include a result that provides bounds for $\mathrm{E}\left[W_{n}^{p}\right]$ for integer $p$, which will be used in the proof of Lemma 4.3.

Proof of Lemma 4.1. Let $p=\lceil\beta\rceil \in\{2,3, \ldots\}$ and $\gamma=\beta / p \in(\beta /(\beta+1), 1]$. Suppose first that $k \in \mathbb{N}$, and define $A_{p}(k)=\left\{\left(j_{1}, \ldots, j_{k}\right) \in \mathbb{N}^{k}: j_{1}+\cdots+j_{k}=p, 0 \leq j_{i}<p\right\}$. Then, for any sequence of nonnegative numbers $\left\{y_{i}\right\}_{i \geq 1}$, we have

$$
\begin{aligned}
\left(\sum_{i=1}^{k} y_{i}\right)^{\beta} & =\left(\sum_{i=1}^{k} y_{i}\right)^{p \gamma} \\
& =\left(\sum_{i=1}^{k} y_{i}^{p}+\sum_{\left(j_{1}, \ldots, j_{k}\right) \in A_{p}(k)}\left(\begin{array}{c}
p \\
j_{1}, \ldots, j_{k}
\end{array}\right) y_{1}^{j_{1}} \cdots y_{k}^{j_{k}}\right)^{\gamma} \\
& \leq \sum_{i=1}^{k} y_{i}^{p \gamma}+\left(\sum_{\left(j_{1}, \ldots, j_{k}\right) \in A_{p}(k)}\left(\begin{array}{c}
p \\
j_{1}, \ldots, j_{k}
\end{array}\right) y_{1}^{j_{1}} \cdots y_{k}^{j_{k}}\right)^{\gamma},
\end{aligned}
$$

where for the last step we used the well-known inequality $\left(\sum_{i=1}^{k} x_{i}\right)^{\gamma} \leq \sum_{i=1}^{k} x_{i}^{\gamma}$ for $0<\gamma \leq 1$ and $x_{i} \geq 0$. We now use the conditional Jensen inequality to obtain

$$
\begin{aligned}
\mathrm{E}[ & \left.\left(\sum_{i=1}^{k} C_{i} Y_{i}\right)^{\beta}-\sum_{i=1}^{k}\left(C_{i} Y_{i}\right)^{\beta}\right] \\
& \left.\leq \mathrm{E}\left[\left(\sum_{\left(j_{1}, \ldots, j_{k}\right) \in A_{p}(k)}\left(\begin{array}{c}
p \\
j_{1}, \ldots, j_{k}
\end{array}\right)\left(C_{1} Y_{1}\right)^{j_{1}} \cdots\left(C_{k} Y_{k}\right)^{j_{k}}\right)^{\gamma}\right] \quad \text { by }(7.1)\right) \\
& \leq \mathrm{E}\left[\left(\mathrm{E}\left[\sum_{\left(j_{1}, \ldots, j_{k}\right) \in A_{p}(k)}\left(\begin{array}{c}
p \\
j_{1}, \ldots, j_{k}
\end{array}\right)\left(C_{1} Y_{1}\right)^{j_{1}} \ldots\left(C_{k} Y_{k}\right)^{j_{k}} \mid C_{1}, \ldots, C_{k}\right]\right)^{\gamma}\right] \\
& =\mathrm{E}\left[\left(\sum_{\left(j_{1}, \ldots, j_{k}\right) \in A_{p}(k)}\left(\begin{array}{c}
p \\
j_{1}, \ldots, j_{k}
\end{array}\right) C_{1}^{j_{1}} \ldots C_{k}^{j_{k}} \mathrm{E}\left[Y_{1}^{j_{1}} \ldots Y_{k}^{j_{k}} \mid C_{1}, \ldots, C_{k}\right]\right)^{\gamma}\right] .
\end{aligned}
$$

Since $\left\{Y_{i}\right\}$ is a sequence of i.i.d. random variables having the same distribution as $Y$, independent of the $C_{i}$ s, we have

$$
\mathrm{E}\left[Y_{1}^{j_{1}} \cdots Y_{k}^{j_{k}} \mid C_{1}, \ldots, C_{k}\right]=\mathrm{E}\left[Y_{1}^{j_{1}} \cdots Y_{k}^{j_{k}}\right]=\|Y\|_{j_{1}}^{j_{1}} \cdots\|Y\|_{j_{k}}^{j_{k}},
$$

where $\|Y\|_{\kappa}=\left(\mathrm{E}\left[|Y|^{\kappa}\right]\right)^{1 / \kappa}$ for $\kappa \geq 1$ and $\|Y\|_{0}:=1$. Since $\|Y\|_{\kappa}$ is increasing for $\kappa \geq 1$, it follows that $\|Y\|_{j_{i}}^{j_{i}} \leq\|Y\|_{p-1}^{j_{i}}$. Hence, $\|Y\|_{j_{1}}^{j_{1}} \cdots\|Y\|_{j_{k}}^{j_{k}} \leq\|Y\|_{p-1}^{p}$, which in turn implies that

$$
\begin{aligned}
\mathrm{E}\left[\left(\sum_{i=1}^{k} C_{i} Y_{i}\right)^{\beta}-\sum_{i=1}^{k}\left(C_{i} Y_{i}\right)^{\beta}\right] & \leq \mathrm{E}\left[\left(\sum_{\left(j_{1}, \ldots, j_{k}\right) \in A_{p}(k)}\left(\begin{array}{c}
p \\
j_{1}, \ldots, j_{k}
\end{array}\right) C_{1}^{j_{1}} \cdots C_{k}^{j_{k}}\|Y\|_{p-1}^{p}\right)^{\gamma}\right] \\
& =\|Y\|_{p-1}^{\beta} \mathrm{E}\left[\left(\left(\sum_{i=1}^{k} C_{i}\right)^{p}-\sum_{i=1}^{k} C_{i}^{p}\right)^{\gamma}\right] \\
& \leq\|Y\|_{p-1}^{\beta} \mathrm{E}\left[\left(\sum_{i=1}^{k} C_{i}\right)^{\beta}\right] .
\end{aligned}
$$

This completes the proof for finite $k$. 
When $k=\infty$, first note that, from the well-known inequality $\left(x_{1}+x_{2}\right)^{\beta} \geq x_{1}^{\beta}+x_{2}^{\beta}$ for $x_{1}, x_{2} \geq 0$ and $\beta>1$, we obtain the monotonicity in $k$ of the following difference:

$$
\left(\sum_{i=1}^{k+1} C_{i} Y_{i}\right)^{\beta}-\sum_{i=1}^{k+1}\left(C_{i} Y_{i}\right)^{\beta} \geq\left(\sum_{i=1}^{k} C_{i} Y_{i}\right)^{\beta}-\sum_{i=1}^{k}\left(C_{i} Y_{i}\right)^{\beta} \geq 0
$$

Hence,

$$
\begin{aligned}
& \mathrm{E}\left[\left(\sum_{i=1}^{\infty} C_{i} Y_{i}\right)^{\beta}-\sum_{i=1}^{\infty}\left(C_{i} Y_{i}\right)^{\beta}\right] \\
& \quad=\lim _{k \rightarrow \infty} \mathrm{E}\left[\left(\left(\sum_{i=1}^{k} C_{i} Y_{i}\right)^{\beta}-\sum_{i=1}^{k}\left(C_{i} Y_{i}\right)^{\beta}\right)\right] \\
& \quad \leq \lim _{k \rightarrow \infty} \mathrm{E}\left[\left(\sum_{\left(j_{1}, \ldots, j_{k}\right) \in A_{p}(k)}\left(\begin{array}{c}
p \\
j_{1}, \ldots, j_{k}
\end{array}\right)\left(C_{1} Y_{1}\right)^{j_{1}} \cdots\left(C_{k} Y_{k}\right)^{j_{k}}\right)^{\gamma}\right] \\
& \quad \leq \lim _{k \rightarrow \infty}\|Y\|_{p-1}^{\beta} \mathrm{E}\left[\left(\sum_{i=1}^{k} C_{i}\right)^{\beta}\right] \\
& =\|Y\|_{p-1}^{\beta} \mathrm{E}\left[\left(\sum_{i=1}^{\infty} C_{i}\right)^{\beta}\right],
\end{aligned}
$$

where (7.2) and (7.3) are justified by monotone convergence.

Proof of Lemma 4.2. We use the well-known inequality $\left(\sum_{i=1}^{k} y_{i}\right)^{\beta} \leq \sum_{i=1}^{k} y_{i}^{\beta}$ for $0<$ $\beta \leq 1, y_{i} \geq 0$, and $k \leq \infty$, to obtain

$$
\begin{aligned}
\mathrm{E}\left[W_{n}^{\beta}\right] & =\mathrm{E}\left[\left(\sum_{i=1}^{N} C_{i} W_{(n-1), i}\right)^{\beta}\right] \\
& \leq \mathrm{E}\left[\sum_{i=1}^{N} C_{i}^{\beta} W_{(n-1), i}^{\beta}\right] \\
& =\mathrm{E}\left[W_{n-1}^{\beta}\right] \rho_{\beta} \quad \text { (by conditioning on } N, C_{i} \text { and Fubini's theorem) } \\
& \leq \rho_{\beta}^{n} \mathrm{E}\left[W_{0}^{\beta}\right] \quad \text { (iterating } n \text { times) } \\
& =\rho_{\beta}^{n} \mathrm{E}\left[Q^{\beta}\right] .
\end{aligned}
$$

Before proving the moment inequality for general $\beta>1$, we will show first the corresponding result for integer moments.

Lemma 7.1. Let $p \in\{2,3, \ldots\}$, and recall that $\rho_{p}=\mathrm{E}\left[\sum_{i=1}^{N} C_{i}^{p}\right], \rho \equiv \rho_{1}$. Suppose that $\mathrm{E}\left[Q^{p}\right]<\infty, \mathrm{E}\left[\left(\sum_{i=1}^{N} C_{i}\right)^{p}\right]<\infty$, and $\rho \vee \rho_{p}<1$. Then, there exists a constant $K_{p}>0$ such that

$$
\mathrm{E}\left[W_{n}^{p}\right] \leq K_{p}\left(\rho \vee \rho_{p}\right)^{n} \quad \text { for all } n \geq 0 .
$$


Proof. We will give an induction proof in $p$. For $p=2$, we have

$$
\begin{aligned}
\mathrm{E}\left[W_{n}^{2}\right] & =\mathrm{E}\left[\left(\sum_{i=1}^{N} C_{i} W_{(n-1), i}\right)^{2}\right] \\
& =\mathrm{E}\left[\sum_{i=1}^{N} C_{i}^{2} W_{(n-1), i}^{2}+\sum_{i \neq j} C_{i} W_{(n-1), i} C_{j} W_{(n-1), j}\right] \\
& =\mathrm{E}\left[W_{n-1}^{2}\right] \mathrm{E}\left[\sum_{i=1}^{N} C_{i}^{2}\right]+\left(\mathrm{E}\left[W_{n-1}\right]\right)^{2} \mathrm{E}\left[\sum_{i \neq j} C_{i} C_{j}\right]
\end{aligned}
$$

(by conditioning on $N, C_{i}$ and Fubini's theorem)

$$
\leq \rho_{2} \mathrm{E}\left[W_{n-1}^{2}\right]+\mathrm{E}\left[\left(\sum_{i=1}^{N} C_{i}\right)^{2}\right]\left(\mathrm{E}\left[W_{n-1}\right]\right)^{2} .
$$

Using the preceding recursion and noting that

$$
\mathrm{E}\left[W_{n-1}\right]=\rho^{n-1} \mathrm{E}[Q],
$$

we obtain

$$
\mathrm{E}\left[W_{n}^{2}\right] \leq \rho_{2} \mathrm{E}\left[W_{n-1}^{2}\right]+K \rho^{2(n-1)},
$$

where $K=\mathrm{E}\left[\left(\sum_{i=1}^{N} C_{i}\right)^{2}\right](\mathrm{E}[Q])^{2}$. Now, iterating (7.4) gives

$$
\begin{aligned}
\mathrm{E}\left[W_{n}^{2}\right] & \leq \rho_{2}\left(\rho_{2} \mathrm{E}\left[W_{n-2}^{2}\right]+K \rho^{2(n-2)}\right)+K \rho^{2(n-1)} \\
& \leq \rho_{2}^{n-1}\left(\rho_{2} \mathrm{E}\left[W_{0}^{2}\right]+K\right)+K \sum_{i=0}^{n-2} \rho_{2}^{i} \rho^{2(n-1-i)} \\
& =\rho_{2}^{n} \mathrm{E}\left[Q^{2}\right]+K \sum_{i=0}^{n-1} \rho_{2}^{i} \rho^{2(n-1-i)} \\
& \leq\left(\rho_{2} \vee \rho\right)^{n} \mathrm{E}\left[Q^{2}\right]+K\left(\rho_{2} \vee \rho\right)^{n} \sum_{i=0}^{n-1}\left(\rho_{2} \vee \rho\right)^{n-i-2} \\
& \leq\left(\mathrm{E}\left[Q^{2}\right]+\frac{K}{\rho_{2} \vee \rho} \sum_{j=0}^{\infty}\left(\rho_{2} \vee \rho\right)^{j}\right)\left(\rho_{2} \vee \rho\right)^{n} \\
& =K_{2}\left(\rho_{2} \vee \rho\right)^{n},
\end{aligned}
$$

which completes the $p=2$ case.

Suppose now that there exists a constant $K_{p-1}>0$ such that $\mathrm{E}\left[W_{n}^{p-1}\right] \leq K_{p-1}\left(\rho_{p-1} \vee \rho\right)^{n}$ for all $n \geq 0$. Then, by Lemmas 4.1 and 4.2, we have

$$
\begin{aligned}
\mathrm{E}\left[W_{n}^{p}\right] & =\mathrm{E}\left[\left(\sum_{i=1}^{N} C_{i} W_{(n-1), i}\right)^{p}-\sum_{i=1}^{N} C_{i}^{p} W_{(n-1), i}^{p}\right]+\mathrm{E}\left[\sum_{i=1}^{N} C_{i}^{p} W_{(n-1), i}^{p}\right] \\
& \leq\left(\mathrm{E}\left[W_{n-1}^{p-1}\right]\right)^{p /(p-1)} \mathrm{E}\left[\left(\sum_{i=1}^{N} C_{i}\right)^{p}\right]+\mathrm{E}\left[\sum_{i=1}^{N} C_{i}^{p} W_{(n-1), i}^{p}\right]
\end{aligned}
$$




$$
\begin{aligned}
& =\left(\mathrm{E}\left[W_{n-1}^{p-1}\right]\right)^{p /(p-1)} \mathrm{E}\left[\left(\sum_{i=1}^{N} C_{i}\right)^{p}\right]+\rho_{p} \mathrm{E}\left[W_{n-1}^{p}\right] \\
& \leq \mathrm{E}\left[\left(\sum_{i=1}^{N} C_{i}\right)^{p}\right]\left(K_{p-1}\right)^{p /(p-1)}\left(\rho_{p-1} \vee \rho\right)^{(n-1) p /(p-1)}+\rho_{p} \mathrm{E}\left[W_{n-1}^{p}\right],
\end{aligned}
$$

where in the second equality we conditioned on $N, C_{i}$ and used Fubini's theorem, and the last inequality corresponds to the induction hypothesis. We then obtain the recursion

$$
\mathrm{E}\left[W_{n}^{p}\right] \leq \rho_{p} \mathrm{E}\left[W_{n-1}^{p}\right]+K\left(\rho_{p-1} \vee \rho\right)^{(n-1) p /(p-1)},
$$

where $K=\mathrm{E}\left[\left(\sum_{i=1}^{N} C_{i}\right)^{p}\right]\left(K_{p-1}\right)^{p /(p-1)}$. Iterating $(7.5)$ as for the $p=2$ case gives

$$
\begin{aligned}
\mathrm{E}\left[W_{n}^{p}\right] & \leq \rho_{p}^{n} \mathrm{E}\left[Q^{p}\right]+K \sum_{i=0}^{n-1} \rho_{p}^{i}\left(\rho_{p-1} \vee \rho\right)^{(n-1-i) p /(p-1)} \\
& \leq\left(\rho_{p} \vee \rho\right)^{n} \mathrm{E}\left[Q^{p}\right]+K \sum_{i=0}^{n-1}\left(\rho_{p} \vee \rho\right)^{((n-1) p-i) /(p-1)} \\
& =\left(\rho_{p} \vee \rho\right)^{n} \mathrm{E}\left[Q^{p}\right]+K\left(\rho_{p} \vee \rho\right)^{n-1} \sum_{i=0}^{n-1}\left(\rho_{p} \vee \rho\right)^{(n-i-1) /(p-1)} \\
& \leq\left(\mathrm{E}\left[Q^{p}\right]+K\left(\rho_{p} \vee \rho\right)^{-1} \sum_{j=0}^{\infty}\left(\rho_{p} \vee \rho\right)^{j /(p-1)}\right)\left(\rho_{p} \vee \rho\right)^{n} \\
& =K_{p}\left(\rho_{p} \vee \rho\right)^{n},
\end{aligned}
$$

where in (7.6) we used the convexity of $\varphi(\beta)=\rho_{\beta}$, i.e. $\rho_{p-1}=\varphi(p-1) \leq \varphi(1) \vee \varphi(p)=$ $\rho \vee \rho_{p}$.

The proof for the general $\beta$-moment, $\beta>1$, is given below.

Proof of Lemma 4.3. Set $p=\lceil\beta\rceil \geq \beta>1$. Then, by Lemmas 4.1 and 4.2,

$$
\begin{aligned}
\mathrm{E}\left[W_{n}^{\beta}\right] & =\mathrm{E}\left[\left(\sum_{i=1}^{N} C_{i} W_{(n-1), i}\right)^{\beta}-\sum_{i=1}^{N} C_{i}^{\beta} W_{(n-1), i}^{\beta}\right]+\mathrm{E}\left[\sum_{i=1}^{N} C_{i}^{\beta} W_{(n-1), i}^{\beta}\right] \\
& \leq\left(\mathrm{E}\left[W_{n-1}^{p-1}\right]\right)^{\beta /(p-1)} \mathrm{E}\left[\left(\sum_{i=1}^{N} C_{i}\right)^{\beta}\right]+\mathrm{E}\left[\sum_{i=1}^{N} C_{i}^{\beta} W_{(n-1), i}^{\beta}\right] \\
& =\left(\mathrm{E}\left[W_{n-1}^{p-1}\right]\right)^{\beta /(p-1)} \mathrm{E}\left[\left(\sum_{i=1}^{N} C_{i}\right)^{\beta}\right]+\rho_{\beta} \mathrm{E}\left[W_{n-1}^{\beta}\right] .
\end{aligned}
$$

By Lemma 7.1,

$$
\begin{aligned}
\mathrm{E}\left[W_{n}^{\beta}\right] & \leq \rho_{\beta} \mathrm{E}\left[W_{n-1}^{\beta}\right]+\mathrm{E}\left[\left(\sum_{i=1}^{N} C_{i}\right)^{\beta}\right]\left(K_{p-1}\right)^{\beta /(p-1)}\left(\rho_{p-1} \vee \rho\right)^{(n-1) \beta /(p-1)} \\
& =\rho_{\beta} \mathrm{E}\left[W_{n-1}^{\beta}\right]+K\left(\rho_{p-1} \vee \rho\right)^{(n-1) \gamma},
\end{aligned}
$$


where $\gamma=\beta /(p-1)>1$. Finally, iterating the preceding bound $n-1$ times gives

$$
\begin{aligned}
\mathrm{E}\left[W_{n}^{\beta}\right] & \leq \rho_{\beta}^{n} \mathrm{E}\left[W_{0}^{\beta}\right]+K \sum_{i=0}^{n-1} \rho_{\beta}^{i}\left(\rho \vee \rho_{p-1}\right)^{\gamma(n-1-i)} \\
& \left.\leq \mathrm{E}\left[W_{0}^{\beta}\right]\left(\rho \vee \rho_{\beta}\right)^{n}+K \sum_{i=0}^{n-1}\left(\rho \vee \rho_{\beta}\right)^{\gamma(n-1-i)+i} \quad \text { (by the convexity of } \varphi(\beta)=\rho_{\beta}\right) \\
& =\mathrm{E}\left[Q^{\beta}\right]\left(\rho \vee \rho_{\beta}\right)^{n}+K\left(\rho \vee \rho_{\beta}\right)^{n-1} \sum_{i=0}^{n-1}\left(\rho \vee \rho_{\beta}\right)^{(\gamma-1) i} \\
& \leq K_{\beta}\left(\rho \vee \rho_{\beta}\right)^{n} .
\end{aligned}
$$

This completes the proof.

\subsection{Linear nonhomogeneous recursion}

In this section we give the proofs of the technical Lemmas 4.6 and 4.7 for the linear recursion.

Proof of Lemma 4.6. Note that the integral is positive since

$$
\mathrm{P}\left(\max _{1 \leq i \leq N} C_{i} R_{i}>t\right)=\mathrm{E}\left[\mathbf{1}\left(\max _{1 \leq i \leq N} C_{i} R_{i}>t\right)\right] \leq \mathrm{E}\left[\sum_{i=1}^{N} \mathbf{1}\left(C_{i} R_{i}>t\right)\right] .
$$

To see that the integral is equal to the expectation involving the $\alpha$-moments, note that

$$
\begin{aligned}
\int_{0}^{\infty}\left(\mathrm{E}\left[\sum_{i=1}^{N} \mathbf{1}\left(C_{i} R_{i}>t\right)\right]-\mathrm{P}\left(\max _{1 \leq i \leq N} C_{i} R_{i}>t\right)\right) t^{\alpha-1} \mathrm{~d} t \\
=\int_{0}^{\infty} \mathrm{E}\left[\sum_{i=1}^{N} \mathbf{1}\left(C_{i} R_{i}>t\right)-\mathbf{1}\left(\max _{1 \leq i \leq N} C_{i} R_{i}>t\right)\right] t^{\alpha-1} \mathrm{~d} t \\
=\mathrm{E}\left[\int_{0}^{\infty}\left(\sum_{i=1}^{N} \mathbf{1}\left(C_{i} R_{i}>t\right)-\mathbf{1}\left(\max _{1 \leq i \leq N} C_{i} R_{i}>t\right)\right) t^{\alpha-1} \mathrm{~d} t\right] \\
=\mathrm{E}\left[\sum_{i=1}^{N} \frac{1}{\alpha}\left(C_{i} R_{i}\right)^{\alpha}-\frac{1}{\alpha}\left(\max _{1 \leq i \leq N} C_{i} R_{i}\right)^{\alpha}\right],
\end{aligned}
$$

(by Fubini's theorem)

where the last equality is justified by the assumption that $\sum_{i=1}^{N}\left(C_{i} R_{i}\right)^{\alpha}<\infty$ a.s.

It now remains to show that the integral (expectation) is finite. To do this, let $\boldsymbol{X}=$ $\left(N, C_{1}, C_{2}, \ldots\right)$. Similar arguments to those used above give

$$
\begin{aligned}
& \int_{0}^{\infty}\left(\mathrm{E}\left[\sum_{i=1}^{N} \mathbf{1}\left(C_{i} R_{i}>t\right)\right]-\mathrm{P}\left(\max _{1 \leq i \leq N} C_{i} R_{i}>t\right)\right) t^{\alpha-1} \mathrm{~d} t \\
& =\int_{0}^{\infty} \mathrm{E}\left[\mathrm{E}\left[\sum_{i=1}^{N} \mathbf{1}\left(C_{i} R_{i}>t\right)-\mathbf{1}\left(\max _{1 \leq i \leq N} C_{i} R_{i}>t\right) \mid \boldsymbol{X}\right]\right] t^{\alpha-1} \mathrm{~d} t \\
& =\mathrm{E}\left[\int_{0}^{\infty} \mathrm{E}\left[\sum_{i=1}^{N} \mathbf{1}\left(C_{i} R_{i}>t\right)-\mathbf{1}\left(\max _{1 \leq i \leq N} C_{i} R_{i}>t\right) \mid \boldsymbol{X}\right] t^{\alpha-1} \mathrm{~d} t\right],
\end{aligned}
$$


where in the last step we used Fubini's theorem. Furthermore,

$$
\begin{gathered}
\mathrm{E}\left[\sum_{i=1}^{N} \mathbf{1}\left(C_{i} R_{i}>t\right)-\mathbf{1}\left(\max _{1 \leq i \leq N} C_{i} R_{i}>t\right) \mid \boldsymbol{X}\right] \\
\quad=\mathrm{E}\left[\mathbf{1}\left(\max _{1 \leq i \leq N} C_{i} R_{i} \leq t\right) \mid \boldsymbol{X}\right]-1+\sum_{i=1}^{N} \mathrm{E}\left[\mathbf{1}\left(C_{i} R_{i}>t\right) \mid \boldsymbol{X}\right] .
\end{gathered}
$$

Note that, given $\boldsymbol{X}$, the random variables $C_{i} R_{i}$ are independent (since the $R \mathrm{~s}$ are), so if we let $\bar{F}(t)=\mathrm{P}(R>t)$ then

$$
\mathrm{E}\left[\mathbf{1}\left(\max _{1 \leq i \leq N} C_{i} R_{i} \leq t\right) \mid \boldsymbol{X}\right]=\prod_{i=1}^{N} \mathrm{E}\left[\mathbf{1}\left(C_{i} R_{i} \leq t\right) \mid \boldsymbol{X}\right]=\prod_{i=1}^{N}\left(1-\bar{F}\left(\frac{t}{C_{i}}\right)\right) .
$$

We now use the inequality $1-x \leq \mathrm{e}^{-x}$ for $x \geq 0$ to obtain

$$
\prod_{i=1}^{N}\left(1-\bar{F}\left(\frac{t}{C_{i}}\right)\right) \leq \exp \left(-\sum_{i=1}^{N} \bar{F}\left(\frac{t}{C_{i}}\right)\right)
$$

Next, let $\delta=\alpha \varepsilon /(1+\varepsilon)$ and set $\beta=\alpha-\delta$. By Markov's inequality,

$$
\sum_{i=1}^{N} \bar{F}\left(\frac{t}{C_{i}}\right) \leq \sum_{i=1}^{N} \mathrm{E}\left[\left(C_{i} R\right)^{\beta} \mid C_{i}\right] t^{-\beta}=t^{-\beta} \mathrm{E}\left[R^{\beta}\right] \sum_{i=1}^{N} C_{i}^{\beta}
$$

Now, define the function $g(x)=\mathrm{e}^{-x}-1+x$ and note that $g(x)$ is increasing for $x \geq 0$. Therefore,

$$
g\left(\sum_{i=1}^{N} \bar{F}\left(\frac{t}{C_{i}}\right)\right) \leq g\left(t^{-\beta} \mathrm{E}\left[R^{\beta}\right] \sum_{i=1}^{N} C_{i}^{\beta}\right) .
$$

This observation combined with the previous derivations gives

$$
\begin{gathered}
\int_{0}^{\infty} \mathrm{E}\left[\sum_{i=1}^{N} \mathbf{1}\left(C_{i} R_{i}>t\right)-\mathbf{1}\left(\max _{1 \leq i \leq N} C_{i} R_{i}>t\right) \mid \boldsymbol{X}\right] t^{\alpha-1} \mathrm{~d} t \\
\leq \int_{0}^{\infty}\left(\mathrm{e}^{-r S_{\beta} t^{-\beta}}-1+r S_{\beta} t^{-\beta}\right) t^{\alpha-1} \mathrm{~d} t
\end{gathered}
$$

where $S_{\beta}=\sum_{i=1}^{N} C_{i}^{\beta}$ and $r=\mathrm{E}\left[R^{\beta}\right]<\infty$. Hence, using the change of variable $u=r S_{\beta} t^{-\beta}$ gives

$$
\int_{0}^{\infty}\left(\mathrm{e}^{-r S_{\beta} t^{-\beta}}-1+r S_{\beta} t^{-\beta}\right) t^{\alpha-1} \mathrm{~d} t=\beta^{-1}\left(r S_{\beta}\right)^{\alpha / \beta} \int_{0}^{\infty}\left(\mathrm{e}^{-u}-1+u\right) u^{-\alpha / \beta-1} \mathrm{~d} u .
$$

Our choice of $\beta=\alpha-\delta$ guarantees that $1<\alpha / \beta=1+\varepsilon<2$. To see that the (nonrandom) 
integral is finite, note that $\mathrm{e}^{-x}-1+x \leq x^{2} / 2$ and $\mathrm{e}^{-x}-1 \leq 0$ for any $x \geq 0$, implying that

$$
\begin{aligned}
\int_{0}^{\infty}\left(\mathrm{e}^{-u}-1+u\right) u^{-\alpha / \beta-1} \mathrm{~d} u & \leq \frac{1}{2} \int_{0}^{1} u^{1-\alpha / \beta} \mathrm{d} u+\int_{1}^{\infty} u^{-\alpha / \beta} \mathrm{d} u \\
& =\frac{1}{2(2-\alpha / \beta)}+\frac{1}{\alpha / \beta-1} \\
& =: K \beta \\
& <\infty .
\end{aligned}
$$

Now, it follows that

$$
\begin{aligned}
\int_{0}^{\infty}\left(\mathrm{E}\left[\sum_{i=1}^{N} \mathbf{1}\left(C_{i} R_{i}>t\right)\right]-\mathrm{P}\left(\max _{1 \leq i \leq N} C_{i} R_{i}>t\right)\right) t^{\alpha-1} \mathrm{~d} t & \leq \mathrm{E}\left[K\left(r S_{\beta}\right)^{\alpha / \beta}\right] \\
& =K r^{\alpha / \beta} \mathrm{E}\left[\left(\sum_{i=1}^{N} C_{i}^{\beta}\right)^{\alpha / \beta}\right] .
\end{aligned}
$$

The last expectation is finite by assumption $(\alpha / \beta=1+\varepsilon)$, which completes the proof.

Proof of Lemma 4.7. Let $S=\sum_{i=1}^{N} C_{i} R_{i}<\infty$ a.s., $p=\lceil\alpha\rceil$, and note that $1 \leq p-1<\alpha$. Then, since $(S+Q)^{\alpha}-S^{\alpha} \geq 0$ and $S^{\alpha}-\sum_{i=1}^{N}\left(C_{i} R_{i}\right)^{\alpha} \geq 0$, we can break the expectation as

$$
\begin{aligned}
\mathrm{E}\left[(S+Q)^{\alpha}-\sum_{i=1}^{N}\left(C_{i} R_{i}\right)^{\alpha}\right] & =\mathrm{E}\left[(S+Q)^{\alpha}-S^{\alpha}\right]+\mathrm{E}\left[\left(\sum_{i=1}^{N} C_{i} R_{i}\right)^{\alpha}-\sum_{i=1}^{N}\left(C_{i} R_{i}\right)^{\alpha}\right] \\
& \leq \mathrm{E}\left[(S+Q)^{\alpha}-S^{\alpha}\right]+\left(\mathrm{E}\left[R^{p-1}\right]\right)^{\alpha /(p-1)} \mathrm{E}\left[\left(\sum_{i=1}^{N} C_{i}\right)^{\alpha}\right],
\end{aligned}
$$

where the inequality is justified by Lemma 4.1. The second expectation is finite since, by assumption, $\mathrm{E}\left[R^{\beta}\right]<\infty$ for any $0<\beta<\alpha$. For the first expectation, we use the inequality

$$
(x+t)^{\kappa} \leq \begin{cases}x^{\kappa}+t^{\kappa}, & 0<\kappa \leq 1, \\ x^{\kappa}+\kappa(x+t)^{\kappa-1} t, & \kappa>1,\end{cases}
$$

for any $x, t \geq 0$. We apply the second inequality $p-1$ times and then the first one to obtain

$$
\begin{aligned}
(x+t)^{\alpha} & \leq x^{\alpha}+\alpha(x+t)^{\alpha-1} t \\
& \leq \cdots \\
& \leq x^{\alpha}+\sum_{i=1}^{p-2} \alpha^{i} x^{\alpha-i} t^{i}+\alpha^{p-1}(x+t)^{\alpha-p+1} t^{p-1} \\
& \leq x^{\alpha}+\alpha^{p} t^{\alpha}+\alpha^{p} \sum_{i=1}^{p-1} x^{\alpha-i} t^{i}
\end{aligned}
$$

Hence, it follows that

$$
\mathrm{E}\left[(S+Q)^{\alpha}-S^{\alpha}\right] \leq \alpha^{p} \mathrm{E}\left[Q^{\alpha}\right]+\alpha^{p} \sum_{i=1}^{p-1} \mathrm{E}\left[S^{\alpha-i} Q^{i}\right]
$$


To see that each of the expectations involving a product of $S$ and $Q$ is finite, let $\boldsymbol{X}=$ $\left(Q, N, C_{1}, C_{2}, \ldots\right)$ and note that, for $i=p-1$,

$$
\begin{aligned}
& \mathrm{E}\left[S^{\alpha-p+1} Q^{p-1}\right] \\
& \quad=\mathrm{E}\left[\mathrm{E}\left[\left(Q^{(p-1) /(\alpha-p+1)} \sum_{j=1}^{N} C_{j} R_{j}\right)^{\alpha-p+1} \mid \boldsymbol{X}\right]\right] \\
& \quad \leq \mathrm{E}\left[\left(\mathrm{E}\left[Q^{(p-1) /(\alpha-p+1)} \sum_{j=1}^{N} C_{j} R_{j} \mid \boldsymbol{X}\right]\right)^{\alpha-p+1}\right] \quad \text { (by Jensen's inequality) } \\
& \quad=(\mathrm{E}[R])^{\alpha-p+1} \mathrm{E}\left[Q^{p-1}\left(\sum_{j=1}^{N} C_{j}\right)^{\alpha-p+1}\right]
\end{aligned}
$$

where the last equality was obtained by using the independence of $\left\{R_{j}\right\}$ and $X$.

For $1 \leq i \leq p-2$, let $q_{i}=\lceil\alpha-i\rceil$ and condition on $Q$ and $\boldsymbol{X}$, respectively, to obtain

$$
\begin{aligned}
\mathrm{E}\left[S^{\alpha-i} Q^{i}\right]= & \mathrm{E}\left[\left(S^{\alpha-i}-\sum_{j=1}^{N}\left(C_{j} R_{j}\right)^{\alpha-i}\right) Q^{i}\right]+\mathrm{E}\left[Q^{i} \sum_{j=1}^{N}\left(C_{j} R_{j}\right)^{\alpha-i}\right] \\
= & \mathrm{E}\left[Q^{i} \mathrm{E}\left[S^{\alpha-i}-\sum_{j=1}^{N}\left(C_{j} R_{j}\right)^{\alpha-i} \mid Q\right]\right]+\mathrm{E}\left[R^{\alpha-i}\right] \mathrm{E}\left[Q^{i} \sum_{j=1}^{N} C_{j}^{\alpha-i}\right] \\
\leq & \mathrm{E}\left[Q^{i}\left(\mathrm{E}\left[R^{q_{i}-1} \mid Q\right]\right)^{(\alpha-i) /\left(q_{i}-1\right)} \mathrm{E}\left[\left(\sum_{j=1}^{N} C_{j}\right)^{\alpha-i} \mid Q\right]\right] \\
& +\mathrm{E}\left[R^{\alpha-i}\right] \mathrm{E}\left[Q^{i}\left(\sum_{j=1}^{N} C_{j}\right)^{\alpha-i}\right] \\
= & \left(\left(\mathrm{E}\left[R^{q_{i}-1}\right]\right)^{(\alpha-i) /\left(q_{i}-1\right)}+\mathrm{E}\left[R^{\alpha-i}\right]\right) \mathrm{E}\left[Q^{i}\left(\sum_{j=1}^{N} C_{j}\right)^{\alpha-i}\right],
\end{aligned}
$$

where for the inequality we used Lemma $4.1(\alpha-i>1)$ and the inequality $\sum_{i=1}^{k} y_{i}^{\beta} \leq$ $\left(\sum_{i=1}^{k} y_{i}\right)^{\beta}$ for any $\beta \geq 1$ and $y_{i} \geq 0$. Note that all the expectations involving $R$ in (7.7) and (7.8) are finite since $\mathrm{E}\left[R^{\beta}\right]<\infty$ for all $0<\beta<\alpha$ by assumption. Next, observe that all the other expectations are of the form $\mathrm{E}\left[Q^{i}\left(\sum_{j=1}^{N} C_{j}\right)^{\alpha-i}\right]$ for $1 \leq i \leq p-1$. To see that these are finite, use Hölder's inequality with $q=\alpha /(\alpha-i)$ and $r=\alpha / i$ to obtain

$$
\begin{aligned}
\mathrm{E}\left[Q^{i}\left(\sum_{j=1}^{N} C_{j}\right)^{\alpha-i}\right] & \leq\left\|\left(\sum_{j=1}^{N} C_{j}\right)^{\alpha-i}\right\|_{q}\left\|Q^{i}\right\|_{r} \\
& =\left(\mathrm{E}\left[\left(\sum_{j=1}^{N} C_{j}\right)^{\alpha}\right]\right)^{1 / q}\left(\mathrm{E}\left[Q^{\alpha}\right]\right)^{1 / r} \\
& <\infty
\end{aligned}
$$




\section{Acknowledgements}

We would like to thank the anonymous referee and Matthias Meiners for their helpful comments.

\section{References}

[1] Aldous, D. J. and Bandyopadhyay, A. (2005). A survey of max-type recursive distributional equation. Ann. Appl. Prob. 15, 1047-1110.

[2] Alsmeyer, G. and Kuhlbusch, D. (2010). Double martingale structure and existence of $\phi$-moments for weighted branching processes. Münster J. Math. 3, 163-212.

[3] Alsmeyer, G. And Meiners, M. (2012). Fixed points of inhomogeneous smoothing transforms. To appear in J. Diff. Equat. Appl.

[4] Alsmeyer, G. And Rösler, U. (2005). A stochastic fixed point equation related to weighted branching with deterministic weights. Electron. J. Prob. 11, 27-56.

[5] Alsmeyer, G. ANd Rösler, U. (2008). A stochastic fixed point equation for weighted minima and maxima. Ann. Inst. H. Poincaré Prob. Statist. 44, 89-103.

[6] Alsmeyer, G., Biggins, J. D. And Meiners, M. (2012). The functional equation of the smoothing transform. To appear in Ann. Prob.

[7] Athreya, K. B., McDonald, D. And Ney, P. (1978). Limit theorems for semi-Markov processes and renewal theory for Markov chains. Ann. Prob. 6, 788-797.

[8] Biggins, J. D. (1977). Martingale convergence in the branching random walk. J. Appl. Prob. 14, 25-37.

[9] Biggins, J. D. And Kyprianou, A. E. (1997). Seneta-Heyde norming in the branching random walk. Ann. Prob. 25, 337-360.

[10] Billingsley, P. (1995). Probability and Measure, 3rd edn. Wiley-Interscience, New York.

[11] Brandt, A. (1986). The stochastic equation $Y_{n+1}=A_{n} Y_{n}+B_{n}$ with stationary coefficients. Adv. Appl. Prob. 18, 211-220.

[12] Durret, R. And Liggett, T. M. (1983). Fixed points of the smoothing transformation. Z. Wahrscheinlichkeitsth. 64, 275-301.

[13] Fill, J. A. And Janson, S. (2001). Approximating the limiting Quicksort distribution. Random Structures Algorithms 19, 376-406.

[14] Goldie, C. M. (1991). Implicit renewal theory and tails of solutions of random equations. Ann. Appl. Prob. 1, 126-166.

[15] GrincevičIus, A. K. (1975). One limit distribution for a random walk on the line. Lithuanian Math. J. 15, 580-589.

[16] Holley, R. And Liggett, T. M. (1981). Generalized potlatch and smoothing processes. Z. Wahrscheinlichkeitsth. $\mathbf{5 5}, 165-195$.

[17] IKsanov, A. M. (2004). Elementary fixed points of the BRW smoothing transforms with infinite number of summands. Stoch. Process. Appl. 114, 27-50.

[18] Jagers, P. AND Rösler, U. (2004). Stochastic fixed points for the maximum. In Mathematics and Computer Science III, eds M. Drmota et al., Birkhäuser, Basel, pp. 325-338.

[19] Jelenković, P. R. and Olvera-Cravioto, M. (2010). Information ranking and power laws on trees. Adv. Appl. Prob. 42, 1057-1093.

[20] Kahane, J.-P. and Peyrière, J. (1976). Sur certaines martingales de Benoit Mandelbrot. Adv. Math. 22, 131145.

[21] Kesten, H. (1973). Random difference equations and renewal theory for product of random matrices. Acta Math. 131, 207-248.

[22] LIU, Q. (1998). Fixed points of a generalized smoothing transformation and applications to the branching random walk. Adv. Appl. Prob. 30, 85-112.

[23] LiU, Q. (2000). On generalized multiplicative cascades. Stoch. Process. Appl. 86, 263-286.

[24] NeIninger, R. AND RÜschendorf, L. (2004). A general limit theorem for recursive algorithms and combinatorial structures. Ann. Appl. Prob. 14, 378-418.

[25] Rösler, U. (1993). The weighted branching process. In Dynamics of Complex and Irregular Systems (Bielefeld, 1991; Bielefeld Encount. Math. Phys. VIII), World Scientific, River Edge, NJ, pp. 154-165.

[26] Rösler, U. AND RÜSCHENDORF, L. (2001). The contraction method for recursive algorithms. Algorithmica 29, $3-33$.

[27] Volkovich, Y. and Litvak, N. (2010). Asymptotic analysis for personalized web search. Adv. Appl. Prob. 42, 577-604.

[28] Waymire, E. C. ANd Williams, S. C. (1995). Multiplicative cascades: dimension spectra and dependence. J. Fourier Anal. Appl. 1995, 589-609. 